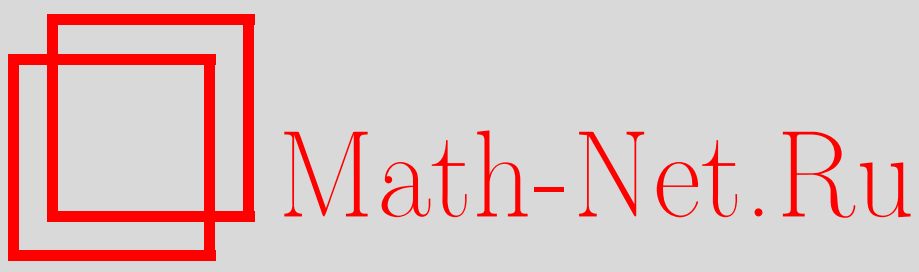

A. E. Gutman, Boolean-valued universe as an algebraic system. ii: intensional hierarchies, Sibirsk. Mat. Zh., 2020, Volume 61, Number 3, 539-571

DOI: https://doi.org/10.33048/smzh.2020.61.305

Use of the all-Russian mathematical portal Math-Net.Ru implies that you have read and agreed to these terms of use http://www . mathnet.ru/eng/agreement

Download details:

IP: 18.209 .158 .208

April 26, 2023, 16:20:23

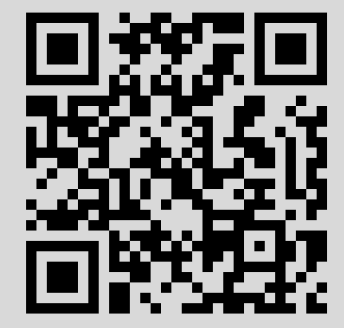


Сибирский математический журнал Май-июнь, 2020. Том 61, № 3

УДК 517.98

\title{
БУЛЕВОЗНАЧНЫЙ УНИВЕРСУМ КАК АЛГЕБРАИЧЕСКАЯ СИСТЕМА. II. ИНТЕНСИОНАЛЬНЫЕ ИЕРАРХИИ
}

\author{
А. Е. Гутман
}

\begin{abstract}
Аннотация. Для булевозначных алгебраических систем теоретико-множественной сигнатуры исследованы понятия транзитивности, регулярности и $\sigma$-регулярности. Введено понятие универсума над произвольной экстенсиональной булевозначной системой и предложено описание структуры универсума посредством различных иерархий. Полученные результаты использованы для доказательства единственности булевозначного универсума с точностью до единственного изоморфизма.
\end{abstract}

DOI 10.33048/smzh.2020.61.305

Ключевые слова: булевозначная алгебраическая система, теория множеств, булевозначный анализ, универсум, кумулятивная иерархия.

\section{Юрию Леонидовичу Ершову \\ в связи с его 80-летием}

Статья продолжает [1] и является второй частью работы, посвященной исследованию булевозначных алгебраических систем теоретико-множественной сигнатуры.

В первой части разработан аппарат частичных элементов булевозначной системы, изучены предикативные булевозначные классы, допускающие квантификацию, предложена интерпретация принципа перемешивания в терминах соединений антицепей частичных элементов, а также установлена взаимосвязь между цикличностью и достижением максимума экстенсиональными булевозначными функциями.

Нумерация параграфов второй части начинается с 7 , продолжая нумерацию статьи [1]. В $\S 7$ исследуется понятие транзитивной булевозначной подсистемы и доказывается аналог леммы А. Леви об абсолютности ограниченных формул для транзитивных моделей. В $\S 8$ синтаксис оценок истинности расширяется кванторами по предикативным булевозначным классам. В $§ 9$ изучается понятие регулярной булевозначной системы и исследуется вопрос о том, для каких булевых алгебр $B$ совпадают классы регулярных и $\sigma$-регулярных $B$-систем. $\mathrm{B} \S 10$ вводится понятие булевозначного универсума над произвольной экстенсиональной булевозначной системой и устанавливается тесная взаимосвязь такого универсума с интенсиональной иерархией - булевозначным аналогом кумулятивной иерархии фон Неймана. В $\S 11$ приводится характеризация классического булевозначного универсума $\mathbb{V}^{(B)}$ как алгебраической системы, доказывается единственность такой системы с точностью до единственного изоморфизма

Работа выполнена при поддержке программы фундаментальных научных исследований CO PAH № I.1.2 (проект № 0314-2019-0005).

(C) 2020 Гутман A. E. 
и для любой полной булевой алгебры $B$ устанавливается логическая независимость условий, фигурирующих в определении $B$-значного универсума. В $\S 12$ предлагается описание структуры булевозначного универсума посредством различных иерархий.

При упоминании любых пунктов параграфов 1-6 неявно подразумевается статья [1]: например, ссылка на п. 2.8 означает ссылку [1, 2.8].

Всюду ниже $B-$ произвольная полная булева алгебра, $\mathbb{N}=\{1,2, \ldots\}-$ множество натуральных чисел, $\omega=\{0,1,2, \ldots\}$ - наименьший бесконечный ординал. Класс всех ординалов обозначается символом Ord, а класс всех предельных ординалов - символом Lim Ord. Кроме того, используются обозначения $\operatorname{Ord}^{\bullet}:=\operatorname{Ord} \cup\{\infty\}$, где $\alpha<\infty$ для всех $\alpha \in$ Ord, и $\operatorname{Lim} \operatorname{Ord}^{\bullet}:=\operatorname{Lim} \operatorname{Ord} \cup\{\infty\}$.

\section{$\S 7$. Транзитивность}

Множество или класс $Y$ называют транзитивным, если

$$
(\forall x, y)(x \in y \in Y \Rightarrow x \in Y) .
$$

Транзитивные классы традиционно используются в теории множеств в качестве моделей фрагментов и модификаций самой теории множеств. При работе с такими моделями полезным инструментом служит лемма А. Леви об абсолютности ограниченных формул. В этом параграфе исследуется понятие транзитивной булевозначной подсистемы и доказывается соответствующий аналог леммы А. Леви.

7.1. Если $Y$ - подсистема $B$-системы $X$ (см. п. 2.8), то запись $\% ~ Y$ можно понимать двояко: как совокупность $(Y \times B) / \sim$ частичных элементов системы $Y$ (см. п. 3.1) или как подкласс $\left\{\left.x\right|_{b}: x \in Y, b \in B\right\} \subset$ \% $X$. Выбор в данном случае непринципиален, поскольку соответствие

$$
\sim_{Y}(y, b) \mapsto \sim_{X}(y, b), \quad y \in Y, b \in B
$$

обеспечивает естественное вложение ${ }^{\%} Y=(Y \times B) / \sim$ в ${ }^{\%} X=(X \times B) / \sim$, и в любом случае можно считать, что ${ }^{\%} Y \subset{ }^{\%} X$.

Аналогичная двусмысленность возникает при интерпретации символа $P \uparrow$ для $P \subset{ }^{\%} Y$ (см. п. 3.5). В рамках системы $X$ или $Y$ булевозначный класс $P \uparrow$ является соответственно функцией $P \uparrow_{X}: X \rightarrow B$ или $P \uparrow_{Y}: Y \rightarrow B$. Но и в этом случае выбор интерпретации не имеет принципиального характера, так как функции $P \uparrow_{X}$ и $P \uparrow_{Y}$ совпадают на $Y$, поэтому истинность включения $[y \in P \uparrow]$ для $y \in Y$ не зависит от системы, в которой она вычисляется. Действительно, если $y \in Y$ и $P=\left\{\left.y_{i}\right|_{b_{i}}: i \in I\right\}$, где $y_{i} \in Y$ и $b_{i} \in B$, то

$$
\begin{aligned}
& {\left[y \in P \uparrow_{Y}\right]_{Y}=P \uparrow_{Y}(y)=\bigvee_{i \in I}\left[y=\left.y_{i}\right|_{b_{i}}\right]_{Y}=\bigvee_{i \in I}\left[y=y_{i}\right]_{Y} \wedge b_{i} } \\
= & \bigvee_{i \in I}\left[y=y_{i}\right]_{X} \wedge b_{i}=\bigvee_{i \in I}\left[y=\left.y_{i}\right|_{b_{i}}\right]_{X}=P \uparrow_{X}(y)=\left[y \in P \uparrow_{X}\right]_{X} .
\end{aligned}
$$

По этой причине вместо $\left[y \in P \uparrow_{Y}\right]_{Y}$ или $\left[y \in P \uparrow_{X}\right]_{X}$ в дальнейшем будем писать просто $[y \in P \uparrow]$ и иногда добавлять индексы $X$ или $Y$ лишь для того, чтобы подчеркнуть, в какой системе производится вычисление. 
Чуть большая аккуратность необходима в обращении с формулой $y \simeq P \uparrow$ для $y \in Y$ и $P \subset{ }^{\%} Y$ (см. п. 3.19). Из соотношения $y \simeq P \uparrow_{X}$ в $X$ вытекает аналогичное соотношение $y \simeq P \uparrow_{Y}$ в $Y$, но, как показано в п. 7.3, обратная импликация имеет место для всех $y \in Y$ и $P \subset{ }^{\%} Y$ в том и только в том случае, когда подсистема $Y \subset X$ транзитивна.

7.2. Пусть $X-$ произвольная $B$-система. Подкласс $Y \subset X$ назовем транзитивным в $X$, если подъем $Y \uparrow$ транзитивен внутри $X$, т. е.

$$
X \vDash(\forall x, y)(x \in y \in Y \uparrow \Rightarrow x \in Y \uparrow) .
$$

Будем говорить, что $Y-$ транзитивная подсистема $X$, и писать $Y \preccurlyeq X$, если $Y$ - непустой транзитивный подкласс $X$, снабженный индуцированными из $X$ интерпретациями (см. п. 2.8).

Следующие свойства подкласса $Y \subset X$ равносильны:

(a) $Y$ транзитивен в $X$;

(b) $(\forall y \in Y) X \vDash(y \subset Y \uparrow)$;

(c) $(\forall x \in X)(\forall y \in Y)[x \in y] \leqslant[x \in Y \uparrow]$;

(d) $(\forall x \in X)(\forall y \in Y)[x \in y]=\bigvee_{z \in Y}[x=z] \wedge[z \in y]$.

$\triangleleft$ Импликации $(\mathrm{a}) \Rightarrow(\mathrm{b}) \Leftrightarrow(\mathrm{c}) \Leftarrow(\mathrm{d})$ очевидны. Импликация (b) $\Rightarrow(\mathrm{a})$ вытекает из леммы $3.18(\mathrm{~d})$.

$(\mathrm{c}) \Rightarrow(\mathrm{d})$. С учетом (c) и 2.4 для любых $x \in X$ и $y \in Y$ имеем

$$
\begin{aligned}
& \bigvee_{z \in Y}[x=z] \wedge[z \in y] \leqslant[x \in y] \leqslant[x \in Y \uparrow] \wedge[x \in y] \\
& =\bigvee_{z \in Y}[x=z] \wedge[x \in y]=\bigvee_{z \in Y}[x=z] \wedge[z \in y] . \triangleright
\end{aligned}
$$

7.3. Пусть $Y$ - подсистема $B$-системы $X$. Следующие утверждения равносильны:

(a) $X \vDash\left(y=P \uparrow_{X}\right) \Leftrightarrow Y \vDash\left(y=P \uparrow_{Y}\right)$ для любого элемента $y \in Y$ и любого класса $P \subset{ }^{\%} Y$;

(b) $X \vDash\left(y=y \Downarrow_{Y} \uparrow_{X}\right)$ для всех $y \in Y$;

(c) $Y \preccurlyeq X$.

$\triangleleft(\mathrm{a}) \Rightarrow(\mathrm{b})$. Согласно лемме 4.3 для всех $y \in Y$ справедливо соотношение $Y \vDash\left(y=y \Downarrow_{Y} \uparrow_{Y}\right)$, а значит, $X \vDash\left(y=y \Downarrow_{Y} \uparrow_{X}\right)$ благодаря (a).

Импликация $(\mathrm{b}) \Rightarrow(\mathrm{c})$ вытекает из 7.2(b) и леммы $3.17(\mathrm{~b})$.

(c) $\Rightarrow$ (a). Пусть $Y \preccurlyeq X, y \in Y$ и $P \subset{ }^{\%} Y$. Из 7.2(b) и леммы 3.17(b) следует, что $X \vDash\left(y \subset Y \uparrow_{X}, P \uparrow_{X} \subset Y \uparrow_{X}\right)$ и тем самым $X \vDash\left(y=y \cap Y \uparrow_{X}, P \uparrow_{X}=P \uparrow_{X} \cap Y \uparrow_{X}\right)$. Поэтому с учетом леммы $3.18(\mathrm{~d})$ имеем

$$
\begin{gathered}
X \vDash\left(y=P \uparrow_{X}\right) \Leftrightarrow X \vDash\left(y \cap Y \uparrow_{X}=P \uparrow_{X} \cap Y \uparrow_{X}\right) \\
\Leftrightarrow X \vDash\left(\forall z \in Y \uparrow_{X}\right)\left(z \in y \Leftrightarrow z \in P \uparrow_{X}\right) \\
\Leftrightarrow(\forall z \in Y) X \vDash\left(z \in y \Leftrightarrow z \in P \uparrow_{X}\right) \Leftrightarrow(\forall z \in Y) Y \vDash\left(z \in y \Leftrightarrow z \in P \uparrow_{Y}\right) \\
\Leftrightarrow Y \vDash(\forall z)\left(z \in y \Leftrightarrow z \in P \uparrow_{Y}\right) \Leftrightarrow Y \vDash\left(y=P \uparrow_{Y}\right) . \triangleright
\end{gathered}
$$

7.4. Пусть $\varphi-$ формула сигнатуры $\{=, \in\}$, и пусть $U-$ переменная, терм или символ класса. Релятивизацией $\varphi$ на $U$ называется формула, полученная из $\varphi$ в результате замены каждого квантора $(\forall x)$ и $(\exists x)$, где $x-$ произвольная переменная, соответствующим квантором $(\forall x \in U)$ и $(\exists x \in U)$ (см. $[2,12.6])$. 
Релятивизация формулы $\varphi$ на $U$ обозначается символом $U \vDash \varphi$ или, более подробно, $U \vDash \varphi(\bar{x})$, где $\bar{x}=x_{1}, \ldots, x_{n}$ - список, содержащий все свободные переменные формулы $\varphi$. Выбор обозначения для релятивизации основан на том факте, что для любых $\bar{x} \in U$ утверждение $U \vDash \varphi(\bar{x})$ равносильно истинности $\varphi(\bar{x})$ в двузначной алгебраической системе $\left(U,=_{U}, \in_{U}\right)$ со стандартными интерпретациями равенства и принадлежности.

7.5. Формулу сигнатуры $\{=, \in\}$ назовем синтаксически ограниченной, если ее можно построить из атомарных формул $x=y$ и $x \in y$ с помощью дизъюнкции, отрицания и добавления кванторов вида $(\exists x \in y)$, где $x$ и $y$ - символы переменных. Говорят, что $\varphi-$ ограниченная формула, если существует такая синтаксически ограниченная формула $\psi$, что $\vdash(\varphi \Leftrightarrow \psi)$, т. е. эквивалентность $\varphi \Leftrightarrow \psi$ доказуема в теории предикатов (является тавтологией). Ограниченные формулы называют также формулами класса $\Sigma_{0}, \Pi_{0}$ или $\Delta_{0}$. Как легко видеть, если в формуле $\varphi$ каждое вхождение квантора имеет вид $(\exists x \in y)$ или $(\forall x \in y)$, то $\varphi$ - ограниченная формула. Примером ограниченной формулы служит релятивизация $U \vDash \varphi$ любой формулы $\varphi$ на переменную $U$.

Приведенное ниже утверждение является следствием классической леммы А. Леви об абсолютности ограниченных формул для транзитивных моделей [3, лемма 34; 2, лемма 12.9].

Лемма. Пусть $\varphi(\bar{y})$ - ограниченная формула со свободными переменными $\bar{y}=y_{1}, \ldots, y_{n}$. Если $X-B$-система и $Y \preccurlyeq X$, то

$$
(\forall \bar{y} \in Y)[\varphi(\bar{y})]_{X}=[\varphi(\bar{y})]_{Y} .
$$

$\triangleleft$ Поскольку лемма А. Леви доказывается в рамках теории предикатов без специальных аксиом, она является тавтологией. Согласно 3.9 заключение этой леммы для транзитивного внутри $X$ булевозначного класса $Y \uparrow_{X}$ истинно в $X$ :

$$
X \vDash\left(\forall \bar{y} \in Y \uparrow_{X}\right)\left(\varphi(\bar{y}) \Leftrightarrow Y \uparrow_{X} \vDash \varphi(\bar{y})\right)
$$

и, в частности,

$$
(\forall \bar{y} \in Y)[\varphi(\bar{y})]_{X}=\left[Y \uparrow_{X} \vDash \varphi(\bar{y})\right]_{X},
$$

где $Y \uparrow_{X} \vDash \varphi(\bar{y})$ - релятивизация $\varphi(\bar{y})$ на $Y \uparrow_{X}$. Равенство

$$
\left[Y \uparrow_{X} \vDash \varphi(\bar{y})\right]_{X}=[\varphi(\bar{y})]_{Y}
$$

легко доказывается индукцией по сложности синтаксически ограниченной формулы $\varphi$. $\triangleright$

7.6. В дальнейшем пригодится более сильная версия леммы А. Леви, задействующая булевозначные классы.

Теорема. Пусть $\varphi-$ ограниченная формула со свободными переменными $y_{1}, \ldots, y_{n}, z_{1}, \ldots, z_{m}$. Если $X-B$-система и $Y \preccurlyeq X$, то

$$
\begin{aligned}
& {\left[\varphi\left(y_{1}, \ldots, y_{n}, \Phi_{1}, \ldots, \Phi_{m}\right)\right]_{X}=\left[\varphi\left(y_{1}, \ldots, y_{n},\left.\Phi_{1}\right|_{Y}, \ldots,\left.\Phi_{m}\right|_{Y}\right)\right]_{Y}} \\
& \text { для любых } y_{1}, \ldots, y_{n} \in Y, \Phi_{1}, \ldots, \Phi_{m} \Subset X, \Phi_{1}, \ldots, \Phi_{m} \leqslant Y \uparrow_{X} .
\end{aligned}
$$

$\triangleleft$ Формулу $\varphi$ назовем абсолютной, если она удовлетворяет условию (12) для любого разбиения списка свободных переменных на две группы $y_{1}, \ldots, y_{n}$ и $z_{1}, \ldots, z_{m}$. (Отметим, что из-за присутствия произвольных булевозначных классов условие (12), вообще говоря, является бесконечным утверждением, см. § 1.) 
Из 3.9 следует, что для доказательства теоремы достаточно установить абсолютность синтаксически ограниченных формул. Воспользуемся индукцией по сложности формулы.

Покажем, что атомарные формулы абсолютны. Пусть $y \in Y, \Phi, \Psi \Subset X$ и $\Phi, \Psi \leqslant Y \uparrow_{X}$. Тогда $X \vDash\left(y \subset Y \uparrow_{X}\right)$ благодаря транзитивности подсистемы $Y$ и, кроме того, $X \vDash\left(\Phi, \Psi \subset Y \uparrow_{X}\right)$ согласно лемме $3.17($ a). Следовательно, с учетом леммы 3.18 имеем

$$
\begin{aligned}
& {[y \in \Phi]_{X}=\Phi(y)=\left.\Phi\right|_{Y}(y)=\left[\left.y \in \Phi\right|_{Y}\right]_{Y}} \\
& {[y=\Phi]_{X}=[(\forall z)(z \in y \Leftrightarrow z \in \Phi)]_{X}=\left[\left(\forall z \in Y \uparrow_{X}\right)(z \in y \Leftrightarrow z \in \Phi)\right]_{X}} \\
& =\bigwedge_{z \in Y}[z \in y]_{X} \Leftrightarrow_{B}[z \in \Phi]_{X}=\bigwedge_{z \in Y}[z \in y]_{Y} \Leftrightarrow_{B}\left[\left.z \in \Phi\right|_{Y}\right]_{Y} \\
& =\left[(\forall z)\left(\left.z \in y \Leftrightarrow z \in \Phi\right|_{Y}\right)\right]_{Y}=\left[y=\left.\Phi\right|_{Y}\right]_{Y} ; \\
& {[\Phi \in y]_{X}=[(\exists z)(z=\Phi \wedge z \in y)]_{X}=\left[\left(\exists z \in Y \uparrow_{X}\right)(z=\Phi \wedge z \in y)\right]_{X}} \\
& =\bigvee_{z \in Y}[z=\Phi]_{X} \wedge[z \in y]_{X}=\bigvee_{z \in Y}\left[z=\left.\Phi\right|_{Y}\right]_{Y} \wedge[z \in y]_{Y} \\
& =\left[(\exists z)\left(z=\left.\Phi\right|_{Y} \wedge z \in y\right)\right]_{Y}=\left[\left.\Phi\right|_{Y} \in y\right]_{Y} ; \\
& {[\Phi=\Psi]_{X}=[(\forall z)(z \in \Phi \Leftrightarrow z \in \Psi)]_{X}=\left[\left(\forall z \in Y \uparrow_{X}\right)(z \in \Phi \Leftrightarrow z \in \Psi)\right]_{X}} \\
& =\bigwedge_{z \in Y}[z \in \Phi]_{X} \Leftrightarrow_{B}[z \in \Psi]_{X}=\bigwedge_{z \in Y}\left[\left.y \in \Phi\right|_{Y}\right]_{Y} \Leftrightarrow_{B}\left[\left.z \in \Psi\right|_{Y}\right]_{Y} \\
& =\left[(\forall z)\left(\left.\left.z \in \Phi\right|_{Y} \Leftrightarrow z \in \Psi\right|_{Y}\right)\right]_{Y}=\left[\left.\Phi\right|_{Y}=\left.\Psi\right|_{Y}\right]_{Y} ; \\
& {[\Phi \in \Psi]_{X}=[(\exists z)(z=\Phi \wedge z \in \Psi)]_{X}=\left[\left(\exists z \in Y \uparrow_{X}\right)(z=\Phi \wedge z \in \Psi)\right]_{X}} \\
& =\bigvee_{z \in Y}[z=\Phi]_{X} \wedge[z \in \Psi]_{X}=\bigvee_{z \in Y}\left[z=\left.\Phi\right|_{Y}\right]_{Y} \wedge\left[\left.z \in \Psi\right|_{Y}\right]_{Y} \\
& =\left[(\exists z)\left(z=\left.\left.\Phi\right|_{Y} \wedge z \in \Psi\right|_{Y}\right)\right]_{Y}=\left[\left.\left.\Phi\right|_{Y} \in \Psi\right|_{Y}\right]_{Y} .
\end{aligned}
$$

Как легко видеть, абсолютность формул сохраняется отрицанием и дизъюнкцией. Остается показать, что из абсолютности формулы $\varphi(x, y, \bar{y}, \bar{z})$ вытекает абсолютность формулы $\psi(y, \bar{y}, \bar{z}):=(\exists x \in y) \varphi(x, y, \bar{y}, \bar{z})$. Действительно, если $y, \bar{y}=y_{1}, \ldots, y_{n} \in Y, \Phi, \bar{\Phi}=\Phi_{1}, \ldots, \Phi_{m} \Subset X, \Phi, \Phi_{1}, \ldots, \Phi_{m} \leqslant Y \uparrow_{X}$, то с учетом соотношений $X \vDash\left(y \subset Y \uparrow_{X}\right), X \vDash\left(\Phi \subset Y \uparrow_{X}\right)$ и абсолютности $\varphi$ имеем

$$
\begin{aligned}
{[\psi(y, \bar{y}, \bar{\Phi})]_{X} } & =[(\exists x)(x \in y \wedge \varphi(x, y, \bar{y}, \bar{\Phi}))]_{X}=\left[\left(\exists x \in Y \uparrow_{X}\right)(x \in y \wedge \varphi(x, y, \bar{y}, \bar{\Phi}))\right]_{X} \\
& =\bigvee_{x \in Y}[x \in y]_{X} \wedge[\varphi(x, y, \bar{y}, \bar{\Phi})]_{X}=\bigvee_{x \in Y}[x \in y]_{Y} \wedge\left[\varphi\left(x, y, \bar{y},\left.\bar{\Phi}\right|_{Y}\right)\right]_{Y} \\
& =\left[(\exists x)\left(x \in y \wedge \varphi\left(x, y, \bar{y},\left.\bar{\Phi}\right|_{Y}\right)\right)\right]_{Y}=\left[\psi\left(y, \bar{y},\left.\bar{\Phi}\right|_{Y}\right)\right]_{Y} ; \\
{[\psi(\Phi, \bar{y}, \bar{\Phi})]_{X} } & =[(\exists x)(x \in \Phi \wedge \varphi(x, \Phi, \bar{y}, \bar{\Phi}))]_{X}=\left[\left(\exists x \in Y \uparrow_{X}\right)(x \in \Phi \wedge \varphi(x, \Phi, \bar{y}, \bar{\Phi}))\right]_{X} \\
& =\bigvee_{x \in Y}[x \in \Phi]_{X} \wedge[\varphi(x, \Phi, \bar{y}, \bar{\Phi})]_{X}=\bigvee_{x \in Y}\left[\left.x \in \Phi\right|_{Y}\right]_{Y} \wedge\left[\varphi\left(x,\left.\Phi\right|_{Y}, \bar{y},\left.\bar{\Phi}\right|_{Y}\right)\right]_{Y} \\
& =\left[(\exists x)\left(\left.x \in \Phi\right|_{Y} \wedge \varphi\left(x,\left.\Phi\right|_{Y}, \bar{y},\left.\bar{\Phi}\right|_{Y}\right)\right)\right]_{Y}=\left[\psi\left(\left.\Phi\right|_{Y}, \bar{y},\left.\bar{\Phi}\right|_{Y}\right)\right]_{Y} . \triangleright
\end{aligned}
$$

7.7. Следствие. Если $B$-система $X$ экстенсиональна и $Y \preccurlyeq X$, то $Y$ экстенсиональна.

$\triangleleft$ Согласно определению 2.7 экстенсиональность $B$-системы $X$ означает $X \vDash(\forall x, y) \varphi(x, y)$, где

$$
\varphi(x, y):=(\forall z \in x)(z \in y) \wedge(\forall z \in y)(z \in x) \Rightarrow x=y .
$$


Таким образом, если $X$ экстенсиональна, то $X \vDash\left(\forall x, y \in Y \uparrow_{X}\right) \varphi(x, y)$, откуда благодаря теореме 7.6 и ограниченности формулы $(\forall x, y \in u) \varphi(x, y)$ следует $Y \vDash\left(\forall x,\left.y \in\left(Y \uparrow_{X}\right)\right|_{Y}\right) \varphi(x, y)$, т. е. $Y \vDash(\forall x, y) \varphi(x, y)$, что равносильно экстенсиональности $Y$. $\triangleright$

7.8. Следствие. Пусть $\varphi\left(y_{1}, \ldots, y_{n}, z_{1}, \ldots, z_{m}\right)$ - произвольная формула. Если $X-B$-система и $Y \preccurlyeq X$, то

$$
\left[Y \uparrow_{X} \vDash \varphi\left(y_{1}, \ldots, y_{n}, \Phi_{1}, \ldots, \Phi_{m}\right)\right]_{X}=\left[\varphi\left(y_{1}, \ldots, y_{n},\left.\Phi_{1}\right|_{Y}, \ldots,\left.\Phi_{m}\right|_{Y}\right)\right]_{Y}
$$

для любых $y_{1}, \ldots, y_{n} \in Y, \Phi_{1}, \ldots, \Phi_{m} \Subset X, \Phi_{1}, \ldots, \Phi_{m} \leqslant Y \uparrow_{X}$, где $Y \uparrow_{X} \vDash \varphi-$ релятивизация $\varphi$ на $Y \uparrow_{X}$.

$\triangleleft \mathrm{C}$ учетом ограниченности формулы $x \vDash \varphi(\bar{y}, \bar{z})$ из теоремы 7.6 следует

$$
\left[Y \uparrow_{X} \vDash \varphi(\bar{y}, \bar{\Phi})\right]_{X}=\left[\left.\left(Y \uparrow_{X}\right)\right|_{Y} \vDash \varphi\left(\bar{y},\left.\bar{\Phi}\right|_{Y}\right)\right]_{Y}=\left[Y \uparrow_{Y} \vDash \varphi\left(\bar{y},\left.\bar{\Phi}\right|_{Y}\right)\right]_{Y}=\left[\varphi\left(\bar{y},\left.\bar{\Phi}\right|_{Y}\right)\right]_{Y} . \triangleright
$$

7.9. Пусть $I$ - непустое множество или класс. Семейство $B$-систем $\left(X_{i}\right)_{i \in I}$ назовем направленным, если для любых $i, j \in I$ имеется индекс $k \in I$ такой, что $X_{i}$ и $X_{j}$ являются подсистемами $X_{k}$. Как легко видеть, на объединении $X:=\bigcup_{i \in I} X_{i}$ такого семейства существует единственная пара функций $=_{X}, \in_{X}: X^{2} \rightarrow B$, которая превращает $X$ в $B$-систему, содержащую все $X_{i}$ в качестве подсистем. Имея в виду это обстоятельство, при рассмотрении какоголибо направленного семейства $B$-систем условимся по умолчанию считать его объединение $B$-системой.

7.10. Лемма. (а) Пусть $Y$ и $Z$ - подсистемы $B$-системы $X$. Если $Z \preccurlyeq X$ и $Z \subset Y$, то $Z \preccurlyeq Y$.

(b) Если $X, Y, Z-B$-системы и $Z \preccurlyeq Y \preccurlyeq X$, то $Z \preccurlyeq X$.

(c) Пусть $\left(X_{i}\right)_{i \in I}-$ непустое семейство подсистем $B$-системы $X$. Если $X_{i} \preccurlyeq$ $X$ для всех $i \in I$, то $\bigcup_{i \in I} X_{i} \preccurlyeq X$.

(d) Пусть $I-$ непустое направленное упорядоченное множество или класс, $\left(X_{i}\right)_{i \in I}-$ семейство $B$-систем и $X_{i} \preccurlyeq X_{j}$ при $i \leqslant j$. Тогда $X_{i} \preccurlyeq \bigcup_{j \in I} X_{j}$ для
любых $i \in I$.

(е) Пусть $\left(X_{\alpha}\right)_{\alpha \in \text { Ord }}-$ семейство $B$-систем, $X_{\alpha} \preccurlyeq X_{\alpha+1}$ для $\alpha \in$ Ord и $\bigcup_{\beta<\alpha} X_{\beta} \preccurlyeq X_{\alpha}$ для $\alpha \in \operatorname{Lim}$ Ord. Тогда $X_{\gamma} \preccurlyeq X_{\beta} \preccurlyeq \bigcup_{\alpha \in \text { Ord }} X_{\alpha}$ для любых $\gamma \leqslant \beta \in$ Ord.

$\triangleleft$ (а) Если $Z \subset Y \subset X, Z \preccurlyeq X, y \in Y$ и $z \in Z$, то $[y \in z] \leqslant\left[y \in Z \uparrow_{X}\right]_{X}=$ $\left[y \in Z \uparrow_{Y}\right]_{Y}$.

(b) Если $Z \preccurlyeq Y \preccurlyeq X$, то с учетом 7.2(d) для всех $x \in X$ и $z \in Z$ имеем

$$
[x \in z]=\bigvee_{y \in Y}[x=y] \wedge[y \in z] \leqslant \bigvee_{y \in Y}[x=y] \wedge[y \in Z \uparrow] \leqslant[x \in Z \uparrow]
$$

(c) Если $x \in X$ и $y \in Y:=\bigcup_{i \in I} X_{i}$, то $y \in X_{i}$ для некоторого индекса $i \in I$, откуда в силу соотношения $X_{i} \preccurlyeq X$ следует, что $[x \in y] \leqslant\left[x \in X_{i} \uparrow\right]_{X} \leqslant[x \in Y \uparrow]_{X}$.

(d) Если $i \in I, x \in X:=\bigcup_{j \in I} X_{j}$ и $y \in X_{i}$, то $x \in X_{j}$ для некоторого индекса $i \leqslant j \in I$, откуда с учетом $X_{i} \preccurlyeq X_{j}$ следует, что $[x \in y] \leqslant\left[x \in X_{i} \uparrow\right]_{X_{j}}=\left[x \in X_{i} \uparrow\right]_{X}$.

(е) Для $0 \neq \alpha \in$ Ord положим $\mathscr{X}_{\alpha}:=\bigcup_{\beta<\alpha} X_{\beta}$ и заметим, что $\mathscr{X}_{\alpha} \preccurlyeq X_{\alpha}$. 
Действительно, при $\alpha \in \operatorname{Lim}$ Ord это соотношение явно фигурирует в условии, а если $\alpha=\alpha_{0}+1$, то с учетом очевидной монотонности семейства $\left(X_{\alpha}\right)_{\alpha \in \text { Ord }}$ имеем $\mathscr{X}_{\alpha}=\bigcup_{\beta<\alpha} X_{\beta}=\bigcup_{\beta \leqslant \alpha_{0}} X_{\beta}=X_{\alpha_{0}} \preccurlyeq X_{\alpha_{0}+1}=X_{\alpha}$.

Индукцией по $\alpha$ покажем, что $X_{\beta} \preccurlyeq X_{\alpha}$ при $\beta<\alpha$. Рассмотрим произвольный ординал $\alpha$, предположим, что

$$
X_{\gamma} \preccurlyeq X_{\beta} \text { при } \gamma<\beta<\alpha,
$$

и установим соотношение $X_{\beta} \preccurlyeq X_{\alpha}$ для всех $\beta<\alpha$. Если $\beta<\alpha$, то согласно (13) и (d) имеем $X_{\beta} \preccurlyeq \mathscr{X}_{\alpha}$, откуда благодаря $\mathscr{X}_{\alpha} \preccurlyeq X_{\alpha}$ и (b) следует $X_{\beta} \preccurlyeq X_{\alpha}$.

Осталось заметить, что с учетом (d) из установленного выше вытекает соотношение $X_{\beta} \preccurlyeq \bigcup_{\alpha \in \text { Ord }} X_{\alpha}$ для всех $\beta \in$ Ord. $\triangleright$

\section{§ 8. Квантификация по булевозначным классам}

В этом параграфе язык оценок истинности расширяется кванторами по предикативным булевозначным классам, устанавливается аналог леммы А. Леви для формул расширенного языка и доказывается принцип максимума для булевозначных классов.

8.1. Пусть $\varphi-$ произвольная формула, $X-B$-система. Условимся записывать в виде $(\forall \Phi \Subset X) \varphi(\Phi)$ и $(\exists \Phi \Subset X) \varphi(\Phi)$ утверждения о том, что $\varphi(\Phi)$ выполняется для любого или соответственно некоторого предикативного булевозначного класса $\Phi \Subset X$. Поскольку предикативные классы допускают квантификацию (см. п. 4.6), рассматриваемые утверждения не являются бесконечными и каждое из них может быть записано одной формулой:

$$
\begin{aligned}
& (\forall \Phi \Subset X) \varphi(\Phi) \Leftrightarrow\left(\forall P \subset{ }^{\%} X\right) \varphi(P \uparrow), \\
& (\exists \Phi \Subset X) \varphi(\Phi) \Leftrightarrow\left(\exists P \subset{ }^{\%} X\right) \varphi(P \uparrow) .
\end{aligned}
$$

Аналогично определяются выражения $\bigwedge_{\Phi \Subset X} \tau(\Phi)$ и $\underset{\Phi \Subset X}{\bigvee} \tau(\Phi)$ для терма $\tau(x)$ :

$$
\bigwedge_{\Phi \Subset X} \tau(\Phi)=\bigwedge_{P \subset \% X} \tau(P \uparrow), \quad \bigvee_{\Phi \Subset X} \tau(\Phi)=\bigvee_{P \subset \% X} \tau(P \uparrow)
$$

8.2. Пусть $\varphi-$ формула, $X-B$-система и $\Phi \Subset X$. В дальнейшем при употреблении выражения $[\varphi(\Phi, \ldots)]_{X}$ или $X \vDash \varphi(\Phi, \ldots)$ подразумевается, что формула $\varphi$ может содержать несколько свободных переменных, некоторые из которых, возможно, заменены символами булевозначных классов, т. е. запись $\varphi(\Phi, \ldots)$ служит сокращением для $\varphi\left(\Phi, y_{1}, \ldots, y_{m}, \Psi_{1}, \ldots, \Psi_{n}\right)$, где $y_{i} \in X$ и $\Psi_{j} \Subset X-$ произвольные наперед заданные элементы и булевозначные классы.

Расширим синтаксис булевозначных оценок кванторами по предикативным булевозначным классам, полагая

$$
\begin{aligned}
{[(\forall \Phi) \varphi(\Phi, \ldots)]_{X} } & :=\bigwedge_{\Phi \Subset X}[\varphi(\Phi, \ldots)]_{X}, \\
{[(\exists \Phi) \varphi(\Phi, \ldots)]_{X} } & :=\bigvee_{\Phi \Subset X}[\varphi(\Phi, \ldots)]_{X} .
\end{aligned}
$$

Как легко видеть, если система $X$ экстенсиональна и удовлетворяет принципу подъема, то кванторы по классам в $X$ равноценны обычным кванторам: $[(\forall \Phi) \varphi(\Phi, \ldots)]_{X}=[(\forall x) \varphi(x, \ldots)]_{X},[(\exists \Phi) \varphi(\Phi, \ldots)]_{X}=[(\exists x) \varphi(x, \ldots)]_{X}$. 
8.3. Следующее утверждение показывает, что в любой $B$-системе булевозначные классы удовлетворяют аналогу принципа максимума (см. п. 6.1).

Если $\varphi-$ формула и $X-B$-система, то функция $\Phi \Subset X \mapsto[\varphi(\Phi, \ldots)] \in B$ достигает максимума, т. е.

$$
(\exists \Psi \Subset X)[\varphi(\Psi, \ldots)]=[(\exists \Phi) \varphi(\Phi, \ldots)] .
$$

В частности,

$$
\begin{aligned}
& X \vDash(\forall \Phi) \varphi(\Phi, \ldots) \Leftrightarrow(\forall \Phi \Subset X) X \vDash \varphi(\Phi, \ldots), \\
& X \vDash(\exists \Phi) \varphi(\Phi, \ldots) \Leftrightarrow(\exists \Phi \Subset X) X \vDash \varphi(\Phi, \ldots) .
\end{aligned}
$$

$\triangleleft$ Положим $b:=[(\exists \Phi) \varphi(\Phi, \ldots)]=\bigvee_{\Phi \Subset X}[\varphi(\Phi, \ldots)]$. По принципу исчерпывания $[4,2.1 .10(1)]$ существуют антицепь $\left(d_{i}\right)_{i \in I} \subset B$ и семейство предикативных классов $\Phi_{i} \Subset X(i \in I)$ такие, что $\bigvee_{i \in I} d_{i}=b$ и $d_{i} \leqslant\left[\varphi\left(\Phi_{i}, \ldots\right)\right]$ для всех $i \in I$. Определим предикативный класс $\Phi: X \rightarrow B$, полагая $\Phi(x):=\bigvee_{i \in I} \Phi_{i}(x) \wedge d_{i}$ для $x \in X$. Тогда для всех $i \in I$ и $x \in X$ справедливо равенство $\Phi_{i}(x) \wedge d_{i}=\Phi(x) \wedge d_{i}$, откуда

$$
\left[\Phi_{i}=\Phi\right]=\left[(\forall x)\left(x \in \Phi_{i} \Leftrightarrow x \in \Phi\right)\right]=\bigwedge_{x \in X}\left(\Phi_{i}(x) \Leftrightarrow_{B} \Phi(x)\right) \geqslant d_{i}
$$

и, следовательно, с учетом 3.9

$$
b=\bigvee_{i \in I} d_{i} \leqslant \bigvee_{i \in I}\left[\varphi\left(\Phi_{i}, \ldots\right)\right] \wedge\left[\Phi_{i}=\Phi\right] \leqslant[\varphi(\Phi, \ldots)] . \triangleright
$$

8.4. Лемма. Пусть $\varphi-$ формула, $X-B$-система и $\Psi \Subset X$. Тогда

$$
[(\forall \Phi \subset \Psi) \varphi(\Phi, \ldots)]=[(\forall \Phi) \varphi(\Phi \cap \Psi, \ldots)]=\bigwedge_{\Phi \Subset X: \Phi \leqslant \Psi}[\varphi(\Phi, \ldots)] .
$$

$B$ частности, следующие свойства булевозначного класса $\Psi$ равносильны:

(a) $X \vDash(\forall \Phi \subset \Psi) \varphi(\Phi, \ldots)$;

(b) $X \vDash(\forall \Phi) \varphi(\Phi \cap \Psi, \ldots)$;

(c) $(\forall \Phi \Subset X) \Phi \leqslant \Psi \Rightarrow X \vDash \varphi(\Phi, \ldots)$.

$\triangleleft$ Для любого предикативного класса $\Phi \Subset X$ определим $\Phi_{0} \Subset X$, полагая $\Phi_{0}(x):=\Phi(x) \wedge \Psi(x)$ для всех $x \in X$. Тогда $\Phi_{0} \leqslant \Psi$ и $\left[\Phi_{0} \subset \Psi\right]=\left[\Phi_{0}=\Phi \cap \Psi\right]=1_{B}$.

Поскольку $\left[\Phi_{0} \subset \Psi \Rightarrow \varphi\left(\Phi_{0}, \ldots\right)\right]=\left[\varphi\left(\Phi_{0}, \ldots\right)\right]=[\varphi(\Phi \cap \Psi, \ldots)]$, имеет место неравенство

$$
\bigwedge_{\Phi \Subset X}[\Phi \subset \Psi \Rightarrow \varphi(\Phi, \ldots)] \leqslant \bigwedge_{\Phi \Subset X}[\varphi(\Phi \cap \Psi, \ldots)] .
$$

Далее, если $\Phi \leqslant \Psi$, то $[\Phi \cap \Psi=\Phi]=1_{B}$ и $[\varphi(\Phi \cap \Psi, \ldots)]=[\varphi(\Phi, \ldots)]$, а значит,

$$
\bigwedge_{\Phi \Subset X}[\varphi(\Phi \cap \Psi, \ldots)] \leqslant \bigwedge_{\Phi \Subset X: \Phi \leqslant \Psi}[\varphi(\Phi \cap \Psi, \ldots)]=\bigwedge_{\Phi \Subset X: \Phi \leqslant \Psi}[\varphi(\Phi, \ldots)] .
$$

Наконец, из соотношений

$$
[\varphi(\Phi \cap \Psi, \ldots) \Rightarrow(\Phi \subset \Psi \Rightarrow \varphi(\Phi, \ldots))]=[(\varphi(\Phi \cap \Psi, \ldots) \wedge \Phi \cap \Psi=\Phi) \Rightarrow \varphi(\Phi, \ldots)]=1_{B}
$$

вытекает неравенство

$$
\left[\varphi\left(\Phi_{0}, \ldots\right)\right]=[\varphi(\Phi \cap \Psi, \ldots)] \leqslant[\Phi \subset \Psi \Rightarrow \varphi(\Phi, \ldots)]
$$

и, следовательно,

$$
\bigwedge_{\Phi \Subset X: \Phi \leqslant \Psi}[\varphi(\Phi, \ldots)] \leqslant \bigwedge_{\Phi \Subset X}[\Phi \subset \Psi \Rightarrow \varphi(\Phi, \ldots)] . \triangleright
$$


8.5. Лемма. Пусть $\varphi-$ ограниченная формула. Если $X-B$-система, $Y \preccurlyeq X, \bar{y}=y_{1}, \ldots, y_{m} \in Y, \Psi, \bar{\Psi}=\Psi_{1}, \ldots, \Psi_{n} \Subset X$ и $\Psi, \bar{\Psi} \leqslant Y \uparrow_{X}$, то

$$
[(\forall \Phi \subset \Psi) \varphi(\Phi, \bar{y}, \bar{\Psi})]_{X}=\left[\left(\left.\forall \Phi \subset \Psi\right|_{Y}\right) \varphi\left(\Phi, \bar{y},\left.\bar{\Psi}\right|_{Y}\right)\right]_{Y} .
$$

$\triangleleft$ Если $\Phi \Subset Y$ и $\Phi \leqslant\left.\Psi\right|_{Y}$, то $\Phi \uparrow_{X} \leqslant \Psi$ в силу леммы 3.7(b) и, кроме того, $\left.\left(\Phi \uparrow_{X}\right)\right|_{Y}=\Phi$. Следовательно, с учетом теоремы 7.6 и леммы 8.4 имеют место следующие соотношения:

$$
\begin{aligned}
{[(\forall \Phi \subset \Psi) \varphi(\Phi, \bar{y}, \bar{\Psi})]_{X} } & =\bigwedge_{\Phi \Subset X: \Phi \leqslant \Psi}[\varphi(\Phi, \bar{y}, \bar{\Psi})]_{X} \\
\leqslant & \bigwedge_{\Phi \Subset Y: \Phi \leqslant\left.\Psi\right|_{Y}}\left[\varphi\left(\Phi \uparrow_{X}, \bar{y}, \bar{\Psi}\right)\right]_{X}=\bigwedge_{\Phi \Subset Y: \Phi \leqslant\left.\Psi\right|_{Y}}\left[\varphi\left(\left.\left(\Phi \uparrow_{X}\right)\right|_{Y}, \bar{y},\left.\bar{\Psi}\right|_{Y}\right)\right]_{Y} \\
= & \bigwedge_{\Phi \Subset Y: \Phi \leqslant\left.\Psi\right|_{Y}}\left[\varphi\left(\Phi, \bar{y},\left.\bar{\Psi}\right|_{Y}\right)\right]_{Y}=\left[\left(\left.\forall \Phi \subset \Psi\right|_{Y}\right) \varphi\left(\Phi, \bar{y},\left.\bar{\Psi}\right|_{Y}\right)\right]_{Y} .
\end{aligned}
$$

Обратное неравенство также обеспечивается теоремой 7.6 и леммой 8.4:

$$
\begin{gathered}
{\left[\left(\left.\forall \Phi \subset \Psi\right|_{Y}\right) \varphi\left(\Phi, \bar{y},\left.\bar{\Psi}\right|_{Y}\right)\right]_{Y}=\bigwedge_{\Phi \Subset Y: \Phi \leqslant\left.\Psi\right|_{Y}}\left[\varphi\left(\Phi, \bar{y},\left.\bar{\Psi}\right|_{Y}\right)\right]_{Y}} \\
\leqslant \bigwedge_{\Phi \Subset X:\left.\Phi\right|_{Y} \leqslant\left.\Psi\right|_{Y}}\left[\varphi\left(\left.\Phi\right|_{Y}, \bar{y},\left.\bar{\Psi}\right|_{Y}\right)\right]_{Y}=\bigwedge_{\Phi \Subset X:\left.\Phi\right|_{Y} \leqslant\left.\Psi\right|_{Y}}[\varphi(\Phi, \bar{y}, \bar{\Psi})]_{X} \\
\leqslant \bigwedge_{\Phi \Subset X: \Phi \leqslant \Psi}[\varphi(\Phi, \bar{y}, \bar{\Psi})]_{X}=[(\forall \Phi \subset \Psi) \varphi(\Phi, \bar{y}, \bar{\Psi})]_{X} . \triangleright
\end{gathered}
$$

\section{$\S 9$. Регулярность}

Аксиома регулярности (фундирования) имеет вид

$$
\rho:=(\forall x) \mu(x),
$$

где

$$
\mu(x):=((\exists y)(y \in x) \Rightarrow(\exists y \in x)(\forall z \in x)(z \notin y))
$$

или, что то же самое,

$$
\mu(x):=(x \neq \varnothing \Rightarrow(\exists y \in x)(y \cap x=\varnothing)) .
$$

Если $\mathbb{V}-$ класс всех множеств и $\left(\mathbb{V}_{\alpha}\right)_{\alpha \in \text { Ord }}-$ кумулятивная иерархия фон Неймана, определяемая по рекурсивному правилу

$$
\begin{array}{ll}
\mathbb{V}_{0}=\varnothing ; & \\
\mathbb{V}_{\alpha+1}=\mathscr{P}\left(\mathbb{V}_{\alpha}\right), \quad \alpha \in \text { Ord } ; \\
\mathbb{V}_{\alpha}=\bigcup_{\beta<\alpha} \mathbb{V}_{\beta}, \quad \alpha \in \text { Lim Ord },
\end{array}
$$

то в рамках теории, полученной из ZFC исключением аксиомы регулярности $\rho$, равенство $\mathbb{V}=\bigcup_{\alpha \in \text { Ord }} \mathbb{V}_{\alpha}$ равносильно $\rho$ (см. $\left.[2, \S 6]\right)$. Кроме того, в этой теории аксиома регулярности равносильна $\sigma$-регулярности - отсутствию такой последовательности $\left(x_{n}\right)_{n \in \mathbb{N}}$, что $x_{n+1} \in x_{n}$ для всех $n \in \mathbb{N}$.

В этом параграфе вводятся и изучаются понятия регулярной и $\sigma$-регулярной булевозначной системы и исследуется вопрос о том, для каких булевых алгебр $B$ классы регулярных и $\sigma$-регулярных $B$-систем совпадают. 
9.1. Пусть $X-B$-система и $\Psi \Subset X$. Будем говорить, что булевозначный класс $\Psi$ регулярен в $X$, если

$$
X \vDash(\forall \Phi \subset \Psi) \mu(\Phi)
$$

(см. (14)), и что $B$-система $X$ регулярна вне подкласса $Y \subset X$, если дополнение $\neg(Y \uparrow)$ булевозначного класса $Y \uparrow$ регулярно в $X$, т. е.

$$
X \vDash(\forall \Phi)(\Phi \cap Y \uparrow=\varnothing \Rightarrow \mu(\Phi)) .
$$

Систему $X$ назовем регулярной, если в $X$ регулярен наибольший булевозначный класс $X \uparrow$, т. е.

$$
X \vDash(\forall \Phi) \mu(\Phi) .
$$

Регулярность системы $X$ очевидным образом связана с истинностью в $X$ аксиомы регулярности $\rho$ :

(a) если $X$ предикативна, то из регулярности $X$ следует истинность $X \vDash \rho$;

(b) если $X$ интенсиональна, то из истинности $X \vDash \rho$ следует регулярность $X$;

(c) если $X$ удовлетворяет принципу подъема, то регулярность $X$ равносильна истинности $X \vDash \rho$.

9.2. Лемма. Следующие свойства $B$-системы $X$ равносильны:

(a) система $X$ не является регулярной;

(b) существует множество $P \subset \% X$, удовлетворяющее следующим условиям:

$$
\begin{aligned}
& (\exists p \in P) \operatorname{dom} p \neq 0_{B}, \\
& (\forall p \in P) \bigvee_{q \in P}[q \in p] \geqslant \operatorname{dom} p ;
\end{aligned}
$$

(c) существует последовательность множеств $P_{n} \subset{ }^{\%} X(n \in \mathbb{N})$, удовлетворяющих следующим условиям:

$$
\begin{aligned}
& P_{n}-\text { антицепь; } \\
& \bigvee_{p \in P_{n}} \operatorname{dom} p=\bigvee_{p \in P_{1}} \operatorname{dom} p \neq 0_{B} ; \\
& \left(\forall p \in P_{n}\right)\left(\forall q \in P_{n+1}\right)\left(\operatorname{dom} p \wedge \operatorname{dom} q \neq 0_{B} \Rightarrow \operatorname{dom} q=[q \in p]\right) ; \\
& \left(\forall p \in P_{n}\right) \bigvee_{q \in P_{n+1}}[q \in p]=\operatorname{dom} p .
\end{aligned}
$$

$\triangleleft(\mathrm{a}) \Rightarrow(\mathrm{b})$. Если система $X$ не является регулярной, то согласно 8.3 и теореме $4.5(\mathrm{a})$ существует множество $P \subset{ }^{*} X$, для которого $X \not \models \mu(P \uparrow)$. Последнее означает, что

$$
b:=[P \uparrow \neq \varnothing] \wedge[(\forall y \in P \uparrow)(\exists z \in P \uparrow)(z \in y)] \neq 0_{B} .
$$

Покажем, что множество $\left\{\left.p\right|_{b}: p \in P\right\}$ удовлетворяет условиям (b). Действительно, с учетом (3)

$$
\left.\bigvee_{p \in P} \operatorname{dom} p\right|_{b}=\bigvee_{p \in P} \operatorname{dom} p \wedge b=[P \uparrow \neq \varnothing] \wedge b=b \neq 0_{B}
$$

Кроме того, согласно лемме 3.18

$$
b \leqslant[(\forall y \in P \uparrow)(\exists z \in P \uparrow)(z \in y)]=\bigwedge_{p \in P} \neg \operatorname{dom} p \vee \bigvee_{q \in P}[q \in p]
$$


Следовательно, для всех $p \in P$

$$
b \leqslant \neg \operatorname{dom} p \vee \bigvee_{q \in P}[q \in p]
$$

и поэтому

$$
\begin{gathered}
\bigvee_{q \in P}\left[\left.q \in p\right|_{b}\right]=\bigvee_{q \in P}[q \in p] \wedge \operatorname{dom} p \wedge b \\
=\left(\neg \operatorname{dom} p \vee \bigvee_{q \in P}[q \in p]\right) \wedge \operatorname{dom} p \wedge b \geqslant \operatorname{dom} p \wedge b=\left.\operatorname{dom} p\right|_{b}
\end{gathered}
$$

(b) $\Rightarrow(\mathrm{c})$. Пусть $P \subset$ \% $X$ удовлетворяет условиям (b). По принципу исчерпывания для каждого $p \in{ }^{\%} P$ из равенства $\bigvee_{q \in P}[q \in p]=\operatorname{dom} p$ следует существование такой антицепи $D(p) \subset{ }^{\%} P$, что $\operatorname{dom} q=[q \in p]$ для всех $q \in D(p)$ и

$\bigvee[q \in p]=\operatorname{dom} p$. По той же причине существует антицепь $P_{1} \subset \%$ такая, что $q \in D(p)$

$$
\bigvee_{p \in P_{1}} \operatorname{dom} p=\bigvee_{p \in P} \operatorname{dom} p \neq 0_{B} .
$$

Определим множества $P_{n} \subset{ }^{\%} P(n \in \mathbb{N})$, полагая

$$
P_{n+1}:=\bigcup_{p \in P_{n}} D(p), \quad n \in \mathbb{N} .
$$

Элементарная проверка показывает, что $P_{n}$ удовлетворяют всем условиям, перечисленным в (c).

$(\mathrm{c}) \Rightarrow\left(\right.$ a). Пусть последовательность $\left(P_{n}\right)_{n \in \mathbb{N}}$ удовлетворяет (с). Положим $P:=\bigcup_{n \in \mathbb{N}} P_{n}$ и покажем, что $X \not \models \mu(P \uparrow)$. Действительно, согласно (3)

$$
[P \uparrow \neq \varnothing]=\bigvee_{p \in P} \operatorname{dom} p \geqslant \bigvee_{p \in P_{1}} \operatorname{dom} p \neq 0_{B},
$$

но с учетом леммы 3.18

$$
\begin{gathered}
{[(\exists y \in P \uparrow)(\forall z \in P \uparrow)(z \notin y)]=[(\exists y \in P \uparrow) \neg(\exists z \in P \uparrow)(z \in y)]} \\
=\bigvee_{p \in P} \operatorname{dom} p \wedge \neg\left(\bigvee_{q \in P} \operatorname{dom} q \wedge[q \in p]\right) \\
=\bigvee_{n \in \mathbb{N}} \bigvee_{p \in P_{n}} \operatorname{dom} p \wedge \neg\left(\bigvee_{m \in \mathbb{N}} \bigvee_{q \in P_{m}} \operatorname{dom} q \wedge[q \in p]\right) \\
\leqslant \bigvee_{n \in \mathbb{N}} \bigvee_{p \in P_{n}} \operatorname{dom} p \wedge \neg\left(\bigvee_{q \in P_{n+1}}[q \in p]\right)=\bigvee_{n \in \mathbb{N}} \bigvee_{p \in P_{n}} \operatorname{dom} p \wedge \neg \operatorname{dom} p=0_{B} .
\end{gathered}
$$

9.3. Следующее утверждение вытекает из леммы 8.5 благодаря ограниченности формулы $\mu(x)$.

Следствие. Пусть $X-B$-система, $Y \preccurlyeq X, \Psi \Subset X$ и $\Psi \leqslant Y \uparrow_{X}$. Класс $\Psi$ регулярен в $X$ тогда и только тогда, когда класс $\left.\Psi\right|_{Y}$ регулярен в $Y$.

9.4. Лемма. Пусть $X$ и $Y-B$-системы, и пусть $Z \subset Y \preccurlyeq X$. Если система $Y$ регулярна вне $Z$, то в $X$ истинно утверждение о регулярности разности $Y \uparrow \backslash Z \uparrow$, т. e.

$$
X \vDash(\forall \Phi \subset Y \uparrow \backslash Z \uparrow) \mu(\Phi) .
$$

$\triangleleft$ Положим $\Psi:=Y \uparrow_{X} \wedge \neg\left(Z \uparrow_{X}\right)$. Регулярность $Y$ вне $Z$ означает, что класс $\neg\left(Z \uparrow_{Y}\right)$ регулярен в $Y$, откуда с учетом следствия 9.3 и соотношений $\Psi \leqslant Y \uparrow_{X}$, $\left.\Psi\right|_{Y}=\neg\left(Z \uparrow_{Y}\right)$ вытекает регулярность класса $\Psi$ в $X$. Остается заметить, что $X \vDash(\Psi=Y \uparrow \backslash Z \uparrow) . \triangleright$ 
9.5. Лемма. Пусть $X$ и $Y-B$-системы, и пусть $Z \subset Y \preccurlyeq X$. Если система $Y$ регулярна вне $Z$, а $X$ регулярна вне $Y$, то $X$ регулярна вне $Z$.

$\triangleleft$ Установим истинность $X \vDash(\forall \Phi)(\Phi \cap Z \uparrow=\varnothing \Rightarrow \mu(\Phi))$ путем «рассуждений внутри $X \gg$ (см. п. 3.10).

Пусть $\Phi \cap Z \uparrow=\varnothing$ и $\Phi \neq \varnothing$. Найдем такой элемент $y \in \Phi$, что $y \cap \Phi=\varnothing$. Если $\Phi \cap Y \uparrow=\varnothing$, то искомый $y$ существует в силу регулярности $\neg Y \uparrow$. Пусть теперь $\Phi \cap Y \uparrow \neq \varnothing$. Поскольку класс $Y \uparrow \backslash Z \uparrow$ регулярен (см. лемму 9.4) и $\varnothing \neq \Phi \cap Y \uparrow \subset Y \uparrow \backslash Z \uparrow$, имеется такой элемент $y \in \Phi \cap Y \uparrow$, что $y \cap \Phi \cap Y \uparrow=\varnothing$. Тогда $y \cap \Phi=y \cap \Phi \cap Y \uparrow=\varnothing$, так как в силу транзитивности $Y \uparrow$ из $y \in Y \uparrow$ следует $y \subset Y \uparrow . \triangleright$

9.6. Следствие. Пусть $X$ и $Y-B$-системы и $Y \preccurlyeq X$. Если система $Y$ регулярна, а $X$ регулярна вне $Y$, то $X$ регулярна.

9.7. Лемма. Если $B$-система $X$ регулярна, то любая подсистема $Y \subset X$ регулярна.

$\triangleleft$ Рассмотрим произвольные семейства $\left(y_{i}\right)_{i \in I} \subset Y$ и $\left(b_{i}\right)_{i \in I} \subset B$ и покажем, что $Y \vDash \mu\left(P \uparrow_{Y}\right)$, где $P:=\left\{\left.y_{i}\right|_{b_{i}}: i \in I\right\}$. Действительно, используя регулярность системы $X$ и лемму 3.18 , заключаем, что

$$
\begin{gathered}
{\left[P \uparrow_{Y} \neq \varnothing\right]_{Y}=\bigvee_{i \in I} b_{i}=\left[P \uparrow_{X} \neq \varnothing\right]_{X} \leqslant\left[\left(\exists y \in P \uparrow_{X}\right)\left(\forall z \in P \uparrow_{X}\right)(z \notin y)\right]_{X}} \\
=\bigvee_{i \in I} \bigwedge_{j \in I}\left(\left[y_{j} \notin y_{i}\right]_{X} \wedge b_{i} \wedge b_{j}\right) \vee \neg b_{j}=\bigvee_{i \in I} \bigwedge_{j \in I}\left(\left[y_{j} \notin y_{i}\right]_{Y} \wedge b_{i} \wedge b_{j}\right) \vee \neg b_{j} \\
=\left[\left(\exists y \in P \uparrow_{Y}\right)\left(\forall z \in P \uparrow_{Y}\right)(z \notin y)\right]_{Y} . \triangleright
\end{gathered}
$$

9.8. Пусть $X-B$-система и $\Psi \Subset X$. Будем говорить, что булевозначный класс $\Psi$ б-регулярен в $X$, если

$$
\bigwedge_{n \in \mathbb{N}}\left[x_{n} \in \Psi\right] \wedge\left[x_{n+1} \in x_{n}\right]=0_{B}
$$

для любой последовательности $\left(x_{n}\right)_{n \in \mathbb{N}} \subset X$. Будем говорить, что $B$-система $X$ $\sigma$-регулярна вне подкласса $Y \subset X$, если дополнение $\neg(Y \uparrow)$ булевозначного класса $Y \uparrow \sigma$-регулярно в $X$, т. е.

$$
\bigwedge_{n \in \mathbb{N}}\left[x_{n} \notin Y \uparrow\right] \wedge\left[x_{n+1} \in x_{n}\right]=0_{B}
$$

для любой последовательности $\left(x_{n}\right)_{n \in \mathbb{N}} \subset X$. Систему $X$ назовем $\sigma$-регулярной, если в $X \sigma$-регулярен наибольший класс $X \uparrow$, т. е.

$$
\bigwedge_{n \in \mathbb{N}}\left[x_{n+1} \in x_{n}\right]=0_{B}
$$

для любой последовательности $\left(x_{n}\right)_{n \in \mathbb{N}} \subset X$.

9.9. Теорема. Пусть $X-B$-система.

(a) Если класс $\Psi \Subset X$ регулярен в $X$, то $\Psi \sigma$-регулярен в $X$.

(b) Если $Y \subset X$ и разность $X \backslash Y$ предциклична, то регулярность и $\sigma$-регулярность $X$ вне $Y$ равносильны.

$\triangleleft$ (а) Пусть класс $\Psi$ регулярен в $X$. Рассмотрим произвольную последовательность $\left(x_{n}\right)_{n \in \mathbb{N}} \subset X$ и покажем, что

$$
b:=\bigwedge_{n \in \mathbb{N}}\left[x_{n} \in \Psi\right] \wedge\left[x_{n+1} \in x_{n}\right]=0_{B} .
$$


Положим $\Phi:=\left\{x_{n}: n \in \mathbb{N}\right\} \uparrow$. Тогда $[\Phi \neq \varnothing]=1_{B}$ и, кроме того, с учетом леммы 3.18

$$
[\Phi \subset \Psi]=[(\forall x \in \Phi)(x \in \Psi)]=\bigwedge_{n \in \mathbb{N}}\left[x_{n} \in \Psi\right] \geqslant b,
$$

откуда благодаря регулярности $\Psi$ в $X$ следует, что $[(\exists y \in \Phi)(\forall z \in \Phi)(z \notin y)] \geqslant b$. С другой стороны, вновь привлекая лемму 3.18 , заключаем:

$$
\begin{aligned}
& {[(\exists y \in \Phi)(\forall z \in \Phi)(z \notin y)]=\bigvee_{n \in \mathbb{N}} \bigwedge_{m \in \mathbb{N}}\left[x_{m} \notin x_{n}\right]} \\
& =\neg \bigwedge_{n \in \mathbb{N}} \bigvee_{m \in \mathbb{N}}\left[x_{m} \in x_{n}\right] \leqslant \neg \bigwedge_{n \in \mathbb{N}}\left[x_{n+1} \in x_{n}\right] \leqslant \neg b .
\end{aligned}
$$

(b) Пусть разность $Z:=X \backslash Y \neq \varnothing$ предциклична, и пусть система $X \sigma$-регулярна вне $Y$. Рассмотрим произвольный класс $\Phi \Subset X$, удовлетворяющий неравенству $\Phi \leqslant \neg(Y \uparrow)$, и покажем, что $X \vDash \mu(\Phi)$, т. е.

$$
b:=[\Phi \neq \varnothing \wedge(\forall y \in \Phi)(y \cap \Phi \neq \varnothing)]=0_{B} .
$$

Предварительно заметим, что для любого булевозначного класса $\Psi \Subset X$

$$
(\exists x \in X)[(\exists y \in \Phi) \Psi(y)]=[x \in \Phi] \wedge[\Psi(x)] .
$$

Действительно, согласно лемме 3.17(d) справедливо соотношение

$$
X \vDash(\Phi \subset X \uparrow \backslash Y \uparrow \subset Z \uparrow),
$$

откуда с учетом лемм 3.18 и 5.11 вытекают равенства

$$
\begin{gathered}
{[(\exists y \in \Phi) \Psi(y)]=[(\exists y \in Z \uparrow)(\Phi(y) \wedge \Psi(y))]} \\
=[(\exists y \in(\operatorname{mix} Z) \uparrow)(\Phi(y) \wedge \Psi(y))]=\bigvee_{y \in \operatorname{mix} Z} \Phi(y) \wedge \Psi(y),
\end{gathered}
$$

причем по теореме 6.5 функция $\Phi \wedge \Psi$ достигает максимума на циклическом классе $\operatorname{mix} Z$.

Согласно (16) имеется такой элемент $x_{1} \in X$, что $\left[x_{1} \in \Phi\right]=[\Phi \neq \varnothing] \geqslant b$. Из $[(\forall y \in \Phi)(y \cap \Phi \neq \varnothing)] \geqslant b$ следует $\left[x_{1} \cap \Phi \neq \varnothing\right] \geqslant b$, т. е. $\left[(\exists y \in \Phi)\left(y \in x_{1}\right)\right] \geqslant b$, а значит, в силу (16) существует элемент $x_{2} \in X$ такой, что $\left[x_{2} \in \Phi\right] \wedge\left[x_{2} \in x_{1}\right] \geqslant b$. «Итерируя» эти рассуждения (а строго говоря, применяя рекурсию и аксиому выбора), получаем последовательность $\left(x_{n}\right)_{n \in \mathbb{N}}$ элементов $X$, удовлетворяющую неравенствам $\left[x_{n} \notin Y \uparrow\right] \wedge\left[x_{n+1} \in x_{n}\right] \geqslant\left[x_{n} \in \Phi\right] \wedge\left[x_{n+1} \in x_{n}\right] \geqslant b$ для всех $n \in \mathbb{N}$. Благодаря $\sigma$-регулярности $X$ вне $Y$ отсюда следует, что $b=0$. $\triangleright$

9.10. Завершим параграф исследованием вопроса о том, для каких полных булевых алгебр $B$ совпадают понятия регулярной и $\sigma$-регулярной $B$-системы.

Говорят, что элемент $c \in B$ вписан (строго вписан) в множество $D \subset B$, и пишут $c \preccurlyeq D(c \prec D)$, если $c \leqslant d(c<d)$ для какого-либо элемента $d \in D$. Говорят, что множество $C \subset B$ вписано (строго вписано) в $D$, и пишут $C \preccurlyeq D$ $(C \prec D)$, если $c \preccurlyeq D(c \prec D)$ для всех $c \in C$. Элемент $c$ или множество $C$ называют вписанным в последовательность $\left(D_{n}\right)_{n \in \mathbb{N}}$ подмножеств $B$, если $c \preccurlyeq D_{n}$ $\left(C \preccurlyeq D_{n}\right)$ для всех $n \in \mathbb{N}$.

Множество $C \subset B$ называется покрытием булевой алгебры $B$, если $\vee C=1_{B}$. Разбиением булевой алгебры называют разбиение единицы, т. е. покрытие, являющееся антицепью. Напомним, что согласно принципу исчерпывания в любое покрытие можно вписать разбиение. 
Теорема $[5, \S 19 ; 6]$. Следующие свойства полной булевой алгебры $B$ равносильны:

(а) для любого множества $I$ и любого семейства $\left(b_{i}^{n}\right)_{i \in I}^{n \in \mathbb{N}}$ элементов $B$ справедливо соотношение

$$
\bigwedge_{n \in \mathbb{N}} \bigvee_{i \in I} b_{i}^{n}=\bigvee_{i \in I^{\mathbb{N}}} \bigwedge_{n \in \mathbb{N}} b_{i(n)}^{n}
$$

(b) в любую последовательность покрытий $B$ можно вписать покрытие;

(c) в любую последовательность разбиений $B$ можно вписать разбиение;

(d) для любой последовательности разбиений $\left(D_{n}\right)_{n \in \mathbb{N}}$ булевой алгебры $B$ и любого ненулевого элемента $a \in B$ существует последовательность $d_{n} \in D_{n}$ $(n \in \mathbb{N})$ такая, что

$$
a \wedge \bigwedge_{n \in \mathbb{N}} d_{n} \neq 0_{B} .
$$

Булева алгебра $B$, удовлетворяющая любому из эквивалентных условий (a)-(d), называется $\omega$-дистрибутивной или $(\omega, \infty)$-дистрибутивной.

Всякая атомная полная булева алгебра $\omega$-дистрибутивна. Пополнение булевой фактор-алгебры $\mathscr{P}(\mathbb{N}) / \mathscr{P}_{\text {fin }}(\mathbb{N})$ является безатомной $\omega$-дистрибутивной булевой алгеброй (см. [6, следствие леммы $5 ; 7$, пример 9]). Классическим примером полной булевой алгебры, не являющейся $\omega$-дистрибутивной, служит булева алгебра классов эквивалентности измеримых по Лебегу подмножеств $\mathbb{R}$.

9.11. Измельчением в булевой алгебре $B$ условимся называть последовательность $\left(C_{n}\right)_{n \in \mathbb{N}}$ подмножеств $B$, удовлетворяющую следующим условиям:

(a) $\vee C_{n}=\vee C_{1} \neq 0_{B}$ для всех $n \in \mathbb{N}$;

(b) $\bigwedge_{n \in \mathbb{N}} c_{n}=0_{B}$ для любой последовательности $c_{n} \in C_{n}(n \in \mathbb{N})$.

Измельчение $\left(C_{n}\right)_{n \in \mathbb{N}}$ назовем разбивающим, если для всех $n \in \mathbb{N}$

(c) $0_{B} \notin C_{n}$;

(d) $C_{n}$ - антицепь;

(e) $C_{n+1} \prec C_{n}$.

9.12. Проверка следующего утверждения не составляет труда.

Лемма. Пусть $\left(C_{n}\right)_{n \in \mathbb{N}}$ - разбивающее измельчение в булевой алгебре $B$, и пусть $X:=\bigcup_{n \in \mathbb{N}} C_{n}$.

(a) Для каждого $x \in X$ существует единственная конечная последовательность элементов $c_{1} \in C_{1}, c_{2} \in C_{2}, \ldots, c_{n} \in C_{n}$ такая, что $c_{1}>c_{2}>\cdots>c_{n}=x$.

(b) Если $n \neq m$, то $C_{n} \cap C_{m}=\varnothing$.

(c) Для всякого $x \in X$ обозначим через $h(x)$ то единственное число $n \in \mathbb{N}$, для которого $x \in C_{n}$. Если $\left(x_{i}\right)_{i \in I} \subset X$ и $\bigvee_{i \in I} h\left(x_{i}\right)=\infty$, то $\bigwedge_{i \in I} x_{i}=0_{B}$.

9.13. Теорема. Следующие свойства полной булевой алгебры $B$ равносильны:

(a) в В существует разбивающее измельчение;

(b) в $B$ существует измельчение;

(c) булева алгебра $B$ не является $\omega$-дистрибутивной.

$\triangleleft$ Импликация $(\mathrm{a}) \Rightarrow(\mathrm{b})$ тривиальна. Импликацию $(\mathrm{b}) \Rightarrow(\mathrm{c})$ легко установить с помощью теоремы 9.10 (d). Покажем, что (c) $\Rightarrow(\mathrm{a})$. 
Если булева алгебра $B$ не является $\omega$-дистрибутивной, то согласно теореме $9.10(\mathrm{~d})$ существуют последовательность разбиений $\left(D_{n}\right)_{n \in \mathbb{N}}$ и ненулевой элемент $a \in B$ такие, что

$$
a \wedge \bigwedge_{n \in \mathbb{N}} d_{n}=0_{B}
$$

для любой последовательности $d_{n} \in D_{n}(n \in \mathbb{N})$. Согласно (17) в последовательность $\left(D_{n}\right)_{n \in \mathbb{N}}$ не вписывается никакой ненулевой элемент $b \leqslant a$, а значит, для всякого такого $b$ можно ввести в рассмотрение натуральное число

$$
m(b):=\min \left\{n \in \mathbb{N}: b \nprec D_{n}\right\} .
$$

Поскольку $\vee D_{m(b)}=1_{B}$ и $b \npreceq D_{m(b)}$, существует такой элемент $d \in D_{m(b)}$, что $0_{B}<b \wedge d<b$. Таким образом, множество

$$
P(b):=\left\{b \wedge d: d \in D_{m(b)}\right\} \backslash\left\{0_{B}\right\}
$$

обладает следующими свойствами:

$$
P(b) \text { - антицепь, } \quad \vee P(b)=b, \quad 0_{B}<c<b \text { для всех } c \in P(b) .
$$

Рекурсивно определим множества $C_{n} \subset B(n \in \mathbb{N})$, полагая

$$
\begin{aligned}
& C_{1}:=P(a) ; \\
& C_{n+1}:=\bigcup_{b \in C_{n}} P(b), \quad n \in \mathbb{N},
\end{aligned}
$$

и установим, что последовательность $\left(C_{n}\right)_{n \in \mathbb{N}}$ является разбивающим измельчением. Условия 9.11(a),(c)-(е) очевидны. Остается обосновать 9.11(b).

Индукцией по $n \in \mathbb{N}$ покажем, что $m(b) \geqslant n$ для всех $b \in C_{n}$. База индукции $n=1$ тривиальна. Предположим, что $m(b) \geqslant n$ при $b \in C_{n}$, рассмотрим произвольный элемент $c \in C_{n+1}$ и установим неравенство $m(c) \geqslant n+1$. По определению $C_{n+1}$ имеет место представление $c=b \wedge d$ для некоторых $b \in C_{n}$, $d \in D_{m(b)}$. Поскольку элемент $b$ вписан в $D_{1}, \ldots, D_{m(b)-1}$, в силу неравенства $c \leqslant b$ это же верно для $c$, а значит, $m(c) \geqslant m(b) \geqslant n$. Кроме того, из $c \npreceq D_{m(c)}$ и $c \leqslant d \in D_{m(b)}$ следует, что $m(c) \neq m(b)$, а значит, $m(c) \geqslant n+1$.

Пусть теперь $c_{n} \in C_{n}$ для всех $n \in \mathbb{N}$. По доказанному выше $m\left(c_{n+1}\right)>n$, откуда $c_{n+1} \preccurlyeq D_{n}$ и, следовательно, $c_{n+1} \leqslant d_{n}$ для некоторой последовательности $d_{n} \in D_{n}(n \in \mathbb{N})$. Привлекая (17), заключаем, что

$$
\bigwedge_{n \in \mathbb{N}} c_{n}=c_{1} \wedge \bigwedge_{n \in \mathbb{N}} c_{n+1} \leqslant a \wedge \bigwedge_{n \in \mathbb{N}} d_{n}=0_{B} . \triangleright
$$

9.14. Теорема. Классы регулярных и $\sigma$-регулярных $B$-систем совпадают тогда и только тогда, когда булева алгебра $B$ является $\omega$-дистрибутивной.

$\triangleleft$ НЕОБходимость. Если булева алгебра $B$ не является $\omega$-дистрибутивной, то согласно теореме 9.13 в $B$ существует разбивающее измельчение $\left(C_{n}\right)_{n \in \mathbb{N}}$. Положим $X:=\bigcup_{n \in \mathbb{N}} C_{n}$. Как и в лемме 9.12(c), для всякого $x \in X$ обозначим через $h(x)$ то единственное число $n \in \mathbb{N}$, для которого $x \in C_{n}$. Превратим $X$ в $B$-систему, полагая для $x, y \in X$

$$
\begin{aligned}
& {[x=y]:= \begin{cases}1_{B}, & \text { если } x=y, \\
0_{B} & \text { в противном случае; }\end{cases} } \\
& {[x \in y]:= \begin{cases}x, & \text { если } h(x)=h(y)+1, \\
0_{B} & \text { в противном случае. }\end{cases} }
\end{aligned}
$$


Установим $\sigma$-регулярность системы $X$, для чего рассмотрим произвольную последовательность $\left(x_{n}\right)_{n \in \mathbb{N}} \subset X$ и покажем, что $\bigwedge_{n \in \mathbb{N}}\left[x_{n+1} \in x_{n}\right]=0_{B}$. Это соотношение очевидно, если $\left[x_{n+1} \in x_{n}\right]=0_{B}$ для какого-либо $n \in \mathbb{N}$. В противном случае $h\left(x_{n+1}\right)=h\left(x_{n}\right)+1$ и $\left[x_{n+1} \in x_{n}\right]=x_{n+1}$ для всех $n \in \mathbb{N}$. Тогда $\bigvee_{\in \mathbb{N}} h\left(x_{n+1}\right)=\infty$, откуда с учетом леммы 9.12(с) следует $n \in \mathbb{N}$

$$
\bigwedge_{n \in \mathbb{N}}\left[x_{n+1} \in x_{n}\right]=\bigwedge_{n \in \mathbb{N}} x_{n+1}=0_{B} .
$$

Система $X$ не является регулярной, так как множество

$$
P:=\left\{\left.x\right|_{x}: x \in X\right\} \subset \% X
$$

удовлетворяет условиям (b) леммы 9.2. Действительно, для всех $n \in \mathbb{N}$ и $x \in C_{n}$

$$
\begin{aligned}
& \bigvee_{y \in X}\left[\left.\left.y\right|_{y} \in x\right|_{x}\right]=x \wedge \bigvee_{y \in X}[y \in x] \wedge y \geqslant x \wedge \bigvee_{y \in C_{n+1}}[y \in x] \wedge y \\
& =x \wedge \bigvee_{y \in C_{n+1}} y=x \wedge \vee C_{n+1}=x \wedge \vee C_{n}=x=\left.\operatorname{dom} x\right|_{x}
\end{aligned}
$$

Достаточность. Пусть $X-\sigma$-регулярная, но не регулярная $B$-система. Рассмотрим множества $P_{n} \subset$ \% $(n \in \mathbb{N})$, удовлетворяющие условиям (с) леммы 9.2, положим

$$
C_{n}:=\left\{\operatorname{dom} p: p \in P_{n}\right\}, \quad n \in \mathbb{N},
$$

и покажем, что последовательность $\left(C_{n}\right)_{n \in \mathbb{N}}$ является измельчением в булевой алгебре $B$ (см. теорему 9.13). Действительно, для всех $n \in \mathbb{N}$

$$
\vee C_{n}=\bigvee_{p \in P_{n}} \operatorname{dom} p=\bigvee_{p \in P_{1}} \operatorname{dom} p=\vee C_{1} \neq 0_{B}
$$

Пусть $c_{n} \in C_{n}$ для всех $n \in \mathbb{N}$. Покажем, что $\bigwedge_{n \in \mathbb{N}} c_{n}=0_{B}$. Последнее соотношение очевидно, если $(\exists n \in \mathbb{N}) c_{n} \wedge c_{n+1}=0_{B}$. Пусть теперь $c_{n} \wedge c_{n+1} \neq 0_{B}$ для всех $n \in \mathbb{N}$. Согласно (18) для каждого $n \in \mathbb{N}$ имеется элемент $p_{n} \in P_{n}$ такой, что $c_{n}=\operatorname{dom} p_{n}$. Поскольку $\operatorname{dom} p_{n} \wedge \operatorname{dom} p_{n+1}=c_{n} \wedge c_{n+1} \neq 0_{B}$, из условий 9.2(c) следует, что $c_{n+1}=\operatorname{dom} p_{n+1}=\left[p_{n+1} \in p_{n}\right]$. Таким образом,

$$
\bigwedge_{n \in \mathbb{N}} c_{n} \leqslant \bigwedge_{n \in \mathbb{N}} c_{n+1}=\bigwedge_{n \in \mathbb{N}}\left[p_{n+1} \in p_{n}\right]=0_{B}
$$

благодаря $\sigma$-регулярности системы $X$. $\triangleright$

\section{$\S 10$. Интенсиональная иерархия}

Кумулятивная иерархия фон Неймана $\left(V_{\alpha}\right)_{\alpha \in \text { Ord }}$ над множеством или классом $V_{0}$ определяется посредством трансфинитной рекурсии

$$
\begin{array}{ll}
V_{\alpha+1}=V_{\alpha} \cup \mathscr{P}\left(V_{\alpha}\right), & \alpha \in \text { Ord; } \\
V_{\alpha}=\bigcup_{\beta<\alpha} V_{\beta}, & \alpha \in \text { Lim Ord. }
\end{array}
$$

В этом параграфе определяется интенсиональная иерархия, служащая аналогом иерархии (19) для булевозначных систем, вводится понятие булевозначного универсума над произвольной экстенсиональной булевозначной системой и устанавливается тесная взаимосвязь такого универсума с соответствующей интенсиональной иерархией. 
10.1. Начнем с характеризации надстройки, представляющей собой булевозначный аналог дискретного шага $V_{\alpha+1}=V_{\alpha} \cup \mathscr{P}\left(V_{\alpha}\right)$ иерархии (19).

Пусть $X$ - произвольная экстенсиональная $B$-система, и пусть $Y$ - подкласс $X$. Введем следующие понятия:

$$
\begin{aligned}
X \text { интенсиональна над } Y & \Leftrightarrow\left(\forall P \subset{ }^{\%} Y\right)(\exists x \in X)(x \simeq P \uparrow) \\
& \Leftrightarrow(\forall \Phi \Subset X: \Phi \leqslant Y \uparrow)(\exists x \in X)(x \simeq \Phi) ; \\
X \text { предикативна над } Y \quad \Leftrightarrow(\forall x \in X \backslash Y)(\exists P \subset \% Y)(x \simeq P \uparrow) & \Leftrightarrow X=Y \cup \mathscr{P}_{X}(Y) ; \\
X \text { отделима над } Y & \Leftrightarrow(\forall x \in X)(\forall z \in X \backslash Y)(x \simeq z \Rightarrow x=z) \\
& \Leftrightarrow\left(\forall x_{1}, x_{2} \in X\right)\left(x_{1} \simeq x_{2}, x_{1} \neq x_{2} \Rightarrow x_{1}, x_{2} \in Y\right) .
\end{aligned}
$$

Будем говорить, что экстенсиональная $B$-система $X$ является надстройкой над подсистемой $Y$, если

(a) $Y \preccurlyeq X$;

(b) $X$ интенсиональна над $Y$;

(c) $X$ предикативна над $Y$;

(d) $X$ отделима над $Y$.

Экстенсиональную $B$-систему $X$ назовем надстройкой над копией $B$-системы $Z$, если $X$ является надстройкой над какой-либо подсистемой, изоморфной $Z$.

10.2. Лемма. Для любой экстенсиональной булевозначной системы $Z$ существует надстройка над копией $Z$.

$\triangleleft$ Рассмотрим произвольную экстенсиональную $B$-систему $Z$, ее изоморфную копию $Y:=\{\varnothing\} \times Z$ и положим (см. п. 4.2)

$$
\mathscr{Y}:=\left\{P \subset{ }^{\%} Y: P \text { насыщено, } \neg(\exists y \in Y)\left(y \simeq P \uparrow_{Y}\right)\right\} .
$$

Заметим, что $Y \cap \mathscr{Y}=\varnothing$. Действительно, по определению 3.1 любое подмножество $P \subset{ }^{\%} Y$ состоит из пар, в то время как всякий элемент произведения $Y=\{\varnothing\} \times Z$ в подходе Куратовского имеет вид $\{\{\varnothing\},\{\varnothing, z\}\}$ и тем самым содержит элемент $\{\varnothing\}$, не являющийся парой.

Положим $X:=Y \cup \mathscr{Y}$ и продолжим на $X^{2}$ интерпретации $=_{Y}, \in_{Y}$, полагая

$$
\begin{array}{lll}
=_{X}(y, z):==_{Y}(y, z), & \in_{X}(y, z):=\in_{Y}(y, z), \\
=_{X}(P, Q):=\left[P \uparrow_{Y}=Q \uparrow_{Y}\right]_{Y}, & \in_{X}(P, Q):=\left[P \uparrow_{Y} \in Q \uparrow_{Y}\right]_{Y}, \\
=_{X}(P, y):=\left[P \uparrow_{Y}=y\right]_{Y}, & \in_{X}(P, y):=\left[P \uparrow_{Y} \in y\right]_{Y}, \\
={ }_{X}(y, P):=\left[y=P \uparrow_{Y}\right]_{Y}, & \in_{X}(y, P):=\left[y \in P \uparrow_{Y}\right]_{Y}
\end{array}
$$

для всех $y, z \in Y$ и $P, Q \in \mathscr{Y}$.

Тот факт, что $\left(X,=_{X}, \in_{X}\right)$ является $B$-системой, устанавливается элементарной проверкой условий, перечисленных в определении 2.1. В большинстве случаев для этой проверки достаточны «синтаксический сахар» 3.8 и истинность в $Y$ пропозициональных аксиом и аксиом равенства (см. п. 3.9). Поясним лишь пять случаев, в трех из которых используется экстенсиональность системы $Y$, а в двух других - очевидное неравенство $[\varphi(x)]_{Y} \leqslant[(\exists x) \varphi(x)]_{Y}$. 
Если $x, y, z \in Y$ и $P, Q \in \mathscr{Y}$, то

$$
\begin{aligned}
& =_{X}(x, P) \wedge=_{X}(P, z)=\left[x=P \uparrow_{Y} \wedge P \uparrow_{Y}=z\right]_{Y} \\
& \quad=\left[(\forall y)\left(y \in x \Leftrightarrow y \in P \uparrow_{Y}\right) \wedge(\forall y)\left(y \in P \uparrow_{Y} \Leftrightarrow y \in z\right)\right]_{Y} \\
& \quad \leqslant[(\forall y)(y \in x \Leftrightarrow y \in z)]_{Y} \leqslant[x=z]_{Y}==_{X}(x, z) ; \\
& \epsilon_{X}(P, y) \wedge={ }_{X}(P, z)=\left[P \uparrow_{Y} \in y \wedge P \uparrow_{Y}=z\right]_{Y} \\
& \quad=\left[(\exists x)\left(P \uparrow_{Y}=x \wedge x \in y\right) \wedge P \uparrow_{Y}=z\right]_{Y} \\
& =\left[(\exists x)\left((\forall u)\left(u \in P \uparrow_{Y} \Leftrightarrow u \in x\right) \wedge x \in y\right) \wedge(\forall u)\left(u \in P \uparrow_{Y} \Leftrightarrow u \in z\right)\right]_{Y} \\
& \leqslant[(\exists x)((\forall u)(u \in z \Leftrightarrow u \in x) \wedge x \in y)]_{Y} \\
& \quad \leqslant[(\exists x)(z=x \wedge x \in y)]_{Y} \leqslant[z \in y]_{Y}=\in_{X}(z, y) ; \\
& \in_{X}(P, Q) \wedge={ }_{X}(P, z)=\left[P \uparrow_{Y} \in Q \uparrow_{Y} \wedge P \uparrow_{Y}=z\right]_{Y} \\
& \quad=\left[(\exists x)\left(P \uparrow_{Y}=x \wedge x \in Q \uparrow_{Y}\right) \wedge P \uparrow_{Y}=z\right]_{Y} \\
& \quad=\left[(\exists x)\left((\forall y)\left(y \in P \uparrow_{Y} \Leftrightarrow y \in x\right) \wedge x \in Q \uparrow_{Y}\right) \wedge(\forall y)\left(y \in P \uparrow_{Y} \Leftrightarrow y \in z\right)\right]_{Y} \\
& \leqslant\left[(\exists x)\left((\forall y)(y \in z \Leftrightarrow y \in x) \wedge x \in Q \uparrow_{Y}\right)\right]_{Y} \\
& \leqslant\left[(\exists x)\left(z=x \wedge x \in Q \uparrow_{Y}\right)\right]_{Y} \leqslant\left[z \in Q \uparrow_{Y}\right]_{Y}=\in_{X}(z, Q) ; \\
& \in_{X}(x, y) \wedge={ }_{X}(x, P)=\left[x \in y \wedge x=P \uparrow_{Y}\right]_{Y} \\
& \leqslant\left[(\exists x)\left(P \uparrow_{Y}=x \wedge x \in y\right)\right]_{Y}=\left[P \uparrow_{Y} \in y\right]_{Y}=\in_{X}(P, y) ; \\
& \in_{X}(x, P) \wedge={ }_{X}(x, Q)=\left[x \in P \uparrow_{Y} \wedge x=Q \uparrow_{Y}\right]_{Y} \\
& \quad \leqslant\left[(\exists x)\left(Q \uparrow_{Y}=x \wedge x \in P \uparrow_{Y}\right)\right]_{Y}=\left[Q \uparrow_{Y} \in P \uparrow_{Y}\right]_{Y}=\in \in_{X}(Q, P) .
\end{aligned}
$$

Покажем, что система $X$ экстенсиональна. Действительно, если $u, v \in X$ и $[\cdot \in u]_{X}=[\cdot \in v]_{X}$, то в каждом из трех случаев

$$
u, v \in Y ; \quad u \in Y, v \in \mathscr{Y} ; \quad u, v \in \mathscr{Y}
$$

имеем

$$
\begin{aligned}
{[u=v]_{X}=\left\{\begin{array}{l}
{[u=v]_{Y}=\bigwedge_{z \in Y}[z \in u]_{Y} \Leftrightarrow_{B}[z \in v]_{Y}} \\
{\left[u=v \uparrow_{Y}\right]_{Y}=\bigwedge_{z \in Y}[z \in u]_{Y} \Leftrightarrow_{B}\left[z \in v \uparrow_{Y}\right]_{Y}} \\
{\left[u \uparrow_{Y}=v \uparrow_{Y}\right]_{Y}=\bigwedge_{z \in Y}\left[z \in u \uparrow_{Y}\right]_{Y} \Leftrightarrow_{B}\left[z \in v \uparrow_{Y}\right]_{Y}}
\end{array}\right\} } \\
=\bigwedge_{z \in Y}[z \in u]_{X} \Leftrightarrow \Leftrightarrow_{B}[z \in v]_{X}=1_{B} .
\end{aligned}
$$

10.1(а). Установим транзитивность подсистемы $Y \subset X$, проверив условие 7.2(с). Пусть $u \in X$ и $v \in Y$. Если $u \in Y$, то $[u \in v]_{X} \leqslant 1_{B}=\left[u \in Y \uparrow_{X}\right]_{X}$, а если $u \in \mathscr{Y}$, то

$$
\begin{gathered}
{[u \in v]_{X}=\left[u \uparrow_{Y} \in v\right]_{Y}=\left[(\exists y)\left(u \uparrow_{Y}=y \wedge y \in v\right)\right]_{Y}} \\
\leqslant\left[(\exists y)\left(u \uparrow_{Y}=y\right)\right]_{Y}=\bigvee_{y \in Y}\left[u \uparrow_{Y}=y\right]_{Y}=\bigvee_{y \in Y}[u=y]_{X}=\left[u \in Y \uparrow_{X}\right]_{X} .
\end{gathered}
$$


В дальнейшем пригодится следующее соотношение в $X$ :

$$
P \simeq P \uparrow_{X} \text { для всех } P \in \mathscr{Y} .
$$

Пусть $P=\left\{\left.y_{i}\right|_{b_{i}}: i \in I\right\} \in \mathscr{Y}$. Покажем, что $[x \in P]_{X}=\left[x \in P \uparrow_{X}\right]_{X}$ для всех $x \in X$. Действительно, если $y \in Y$, то $[y \in P]_{X}=\left[y \in P \uparrow_{Y}\right]_{Y}=\left[y \in P \uparrow_{X}\right]_{X}$ (см. пп. 7.1, 7.6), а если $Q \in \mathscr{Y}$, то с учетом леммы 3.16

$$
\begin{gathered}
{[Q \in P]_{X}=\left[Q \uparrow_{Y} \in P \uparrow_{Y}\right]_{Y}=\bigvee_{p \in P}\left[Q \uparrow_{Y}=p\right]_{Y}} \\
=\bigvee_{i \in I}\left[Q \uparrow_{Y}=y_{i}\right]_{Y} \wedge b_{i}=\bigvee_{i \in I}\left[Q=y_{i}\right]_{X} \wedge b_{i}=\left[Q \in P \uparrow_{X}\right]_{X} .
\end{gathered}
$$

10.1(b). Рассмотрим подмножество $P \subset{ }^{\%} Y$ и покажем, что $x \simeq P \uparrow_{X}$ для некоторого элемента $x \in X$.

Пусть $\bar{P}:=P \uparrow_{Y} \Downarrow_{Y} \subset{ }^{\%} Y$ - насыщенная оболочка $P$ в системе $Y$ (см. п. 4.4). Поскольку $P \subset \bar{P} \subset P \uparrow_{X} \Downarrow_{X}$, имеем $P \uparrow_{X} \leqslant \bar{P} \uparrow_{X} \leqslant P \uparrow_{X} \Downarrow_{X} \uparrow_{X}$ и, следовательно, $P \uparrow_{X}=\bar{P} \uparrow_{X}$ в силу равенства $P \uparrow_{X} \Downarrow_{X} \uparrow_{X}=P \uparrow_{X}$ (см. лемму 4.3). Если $y \simeq \bar{P} \uparrow_{Y}$ в $Y$ для некоторого элемента $y \in Y$, то $y \simeq \bar{P}_{X}$ в $X$ согласно 7.3, а значит, $x:=y \simeq \bar{P} \uparrow_{X}=P \uparrow_{X}$. Если же $\neg(\exists y \in Y)\left(y \simeq \bar{P} \uparrow_{Y}\right)$, то $\bar{P} \in \mathscr{Y}$ и в этом случае $x:=\bar{P} \simeq \bar{P} \uparrow_{X}=P \uparrow_{X}$ благодаря (20).

Утверждение 10.1(с) является прямым следствием (20).

10.1(d). Пусть $y \in Y$ и $P \in \mathscr{Y}$. По определению $\mathscr{Y}$ имеем $y \not f \uparrow_{Y}$ в $Y$, откуда согласно 7.3 следует, что $y \not f P \uparrow_{X}$ в $X$, а значит, $y \neq P$ в силу (20).

Пусть теперь $P, Q \in \mathscr{Y}$ и $P \simeq Q$. Из (20) вытекает равенство $P \uparrow_{X}=Q \uparrow_{X}$. Тогда $P \uparrow_{Y}=\left.\left(P \uparrow_{X}\right)\right|_{Y}=\left.\left(Q \uparrow_{X}\right)\right|_{Y}=P \uparrow_{Y}$, а поскольку $P$ и $Q-$ насыщенные подмножества $\%$, согласно лемме 4.2 имеем $P=P \uparrow_{Y} \Downarrow_{Y}=Q \uparrow_{Y} \Downarrow_{Y}=Q . \triangleright$

10.3. Лемма. (а) Если $B$-система $X$ является надстройкой над подсистемой $Y \subset X, f: X \leftrightarrow_{B} X$ и $\left.f\right|_{Y}=\operatorname{id}_{Y}$, то $f=\operatorname{id}_{X}$.

(b) Если $B$-системы $X$ и $X^{\prime}$ являются надстройками над подсистемами $Y \subset X$ и $Y^{\prime} \subset X^{\prime}$, то любой изоморфизм $f: Y \leftrightarrow_{B} Y^{\prime}$ продолжается до единственного изоморфизма $\bar{f}: X \leftrightarrow_{B} X^{\prime} . B$ частности, надстройка над копией булевозначной системы единственна с точностью до изоморфизма.

$\triangleleft$ (а) Предварительно заметим, что из экстенсиональности системы $X$ и условия 10.1(c) вытекает следующее соотношение:

$$
\left(\forall x_{1}, x_{2} \in X \backslash Y\right) \quad\left[x_{1}=x_{2}\right]_{X}=\bigwedge_{y \in Y}\left[y \in x_{1} \Leftrightarrow y \in x_{2}\right]_{X} .
$$

Действительно, для любого элемента $x \in X \backslash Y$ имеется подмножество $P \subset{ }^{\%} Y$ такое, что $X \vDash\left(x=P \uparrow_{X}\right)$, откуда с учетом истинности $X \vDash\left(P \uparrow_{X} \subset Y \uparrow_{X}\right)$ (см. лемму $3.17(\mathrm{~b}))$ вытекает $X \vDash\left(x \subset Y \uparrow_{X}\right)$. Следовательно,

$$
X \vDash\left(x_{1}=x_{2} \Leftrightarrow\left(\forall y \in Y \uparrow_{X}\right)\left(y \in x_{1} \Leftrightarrow y \in x_{2}\right)\right)
$$

для всех $x_{1}, x_{2} \in X \backslash Y$. Остается сослаться на лемму 3.18.

Пусть $X, Y$ и $f$ удовлетворяют условиям, перечисленным в (а). Рассмотрим произвольный элемент $x \in X \backslash Y$ и покажем, что $f(x)=x$. Учитывая (21) и равенства $f(y)=y$ для $y \in Y$, заключаем:

$$
\begin{gathered}
{[f(x)=x]_{X}=\bigwedge_{y \in Y}[y \in f(x) \Leftrightarrow y \in x]_{X}} \\
=\bigwedge_{y \in Y}[f(y) \in f(x)]_{X} \Leftrightarrow_{B}[y \in x]_{X}=\bigwedge_{y \in Y}[y \in x]_{X} \Leftrightarrow_{B}[y \in x]_{X}=1_{B} .
\end{gathered}
$$


Таким образом, $f(x) \simeq x$, откуда в силу $10.1(\mathrm{~d})$ вытекает равенство $f(x)=x$, поскольку $x \notin Y$.

(b) Единственность продолжения $\bar{f}$ следует из (а). Покажем существование. Рассмотрим произвольные экстенсиональные $B$-системы $X$ и $X^{\prime}$, являющиеся надстройками над подсистемами $Y \subset X$ и $Y^{\prime} \subset X^{\prime}$, и изоморфизм $f: Y \leftrightarrow_{B} Y^{\prime}$. Согласно 10.1(c) существует семейство подмножеств $P_{x} \subset{ }^{\%} Y$ таких, что $x \simeq P_{x} \uparrow_{X}$ для всех $x \in X \backslash Y$. Для $x \in X \backslash Y$ положим $P_{x}^{\prime}:=f^{\%}\left(P_{x}\right) \subset{ }^{*} Y^{\prime}$ (см. п. 3.15). В силу 10.1(b) имеется функция $g: X \backslash Y \rightarrow X^{\prime}$, удовлетворяющая соотношению $g(x) \simeq P_{x}^{\prime} \uparrow_{X^{\prime}}$ для всех $x \in X \backslash Y$. Определим $\bar{f}: X \rightarrow X^{\prime}$, полагая

$$
\bar{f}(x):= \begin{cases}f(x), & \text { если } x \in Y, \\ g(x), & \text { если } x \in X \backslash Y,\end{cases}
$$

и покажем, что $\bar{f}: X \leftrightarrow_{B} X^{\prime}$. Ради удобства введем обозначение $x^{\prime}:=\bar{f}(x) \in X^{\prime}$ для $x \in X$. Таким образом, $f: y \mapsto y^{\prime}-$ изоморфизм $Y$ на $Y^{\prime}$, и для всех $x \in X \backslash Y$

$$
\begin{array}{ll}
P_{x} \subset{ }^{\%} Y, & P_{x}^{\prime}=f^{\%}\left(P_{x}\right) \subset{ }^{\%} Y^{\prime}, \\
x \simeq P_{x} \uparrow_{X}, & x^{\prime} \simeq P_{x}^{\prime} \uparrow_{X^{\prime}} .
\end{array}
$$

Покажем, что функция $\bar{f}$ сохраняет истинность атомарных формул. Пусть $\varphi\left(x_{1}, x_{2}, y_{1}, y_{2}\right)$ - любая из (ограниченных) формул $x_{1}=x_{2}, x_{1} \in x_{2}, x_{1}=y_{1}$, $x_{1} \in y_{1}, y_{1} \in x_{1}, y_{1}=y_{2}, y_{1} \in y_{2}$. Тогда согласно лемме 3.15 и теореме 7.6 для всех $x_{1}, x_{2} \in X \backslash Y$ и $y_{1}, y_{2} \in Y$ имеем

$$
\begin{gathered}
{\left[\varphi\left(x_{1}, x_{2}, y_{1}, y_{2}\right)\right]_{X}=\left[\varphi\left(P_{x_{1}} \uparrow_{X}, P_{x_{2}} \uparrow_{X}, y_{1}, y_{2}\right)\right]_{X}} \\
=\left[\varphi\left(P_{x_{1}} \uparrow_{Y}, P_{x_{2}} \uparrow_{Y}, y_{1}, y_{2}\right)\right]_{Y}=\left[\varphi\left(P_{x_{1}}^{\prime} \uparrow_{Y^{\prime}}, P_{x_{2}}^{\prime} \uparrow_{Y^{\prime}}, y_{1}^{\prime}, y_{2}^{\prime}\right)\right]_{Y^{\prime}} \\
=\left[\varphi\left(P_{x_{1}}^{\prime} \uparrow_{X^{\prime}}, P_{x_{2}}^{\prime} \uparrow_{X^{\prime}}, y_{1}^{\prime}, y_{2}^{\prime}\right)\right]_{X^{\prime}}=\left[\varphi\left(x_{1}^{\prime}, x_{2}^{\prime}, y_{1}^{\prime}, y_{2}^{\prime}\right)\right]_{X^{\prime}} .
\end{gathered}
$$

Покажем, что функция $\bar{f}: X \rightarrow X^{\prime}$ инъективна. Инъективность $\bar{f}$ на $Y$ обеспечивается инъективностью изоморфизма $f: Y \rightarrow Y^{\prime}$. Если же хотя бы один из элементов $x_{1}, x_{2} \in X$ не принадлежит $Y$, то с учетом равенства $\left[x_{1}=x_{2}\right]_{X}=$ $\left[x_{1}^{\prime}=x_{2}^{\prime}\right]_{X^{\prime}}($ см. $(22))$ из $x_{1}^{\prime}=x_{2}^{\prime}$ следует $x_{1} \simeq x_{2}$ и тогда $x_{1}=x_{2}$ согласно $10.1(\mathrm{~d})$.

Наконец, установим сюръективность функции $\bar{f}: X \rightarrow X^{\prime}$. Рассмотрим произвольный элемент $z \in X^{\prime}$ и покажем, что $z=x^{\prime}$ для некоторого $x \in X$. Если $z \in Y^{\prime}$, то нужный элемент $x \in X$ существует благодаря сюръективности изоморфизма $f: Y \rightarrow Y^{\prime}$. Пусть $z \in X^{\prime} \backslash Y^{\prime}$. Согласно 10.1(с) имеется подмножество $P^{\prime} \subset{ }^{\prime} Y^{\prime}$, для которого $z \simeq P^{\prime} \uparrow_{X^{\prime}}$. Положим $P:=\left(f^{\%}\right)^{-1}\left(P^{\prime}\right) \subset Y$. В силу 10.1 (b) существует элемент $x \in X$, удовлетворяющий соотношению $x \simeq P \uparrow_{X}$. Если $x \in Y$, то с учетом 7.3 и леммы 3.15 имеют место импликации

$$
x \simeq P \uparrow_{X} \Rightarrow x \simeq P \uparrow_{Y} \Rightarrow x^{\prime} \simeq P^{\prime} \uparrow_{Y^{\prime}} \Rightarrow x^{\prime} \simeq P^{\prime} \uparrow_{X^{\prime}} \Rightarrow x^{\prime} \simeq z,
$$

приводящие к противоречию с условием 10.1(d). Следовательно, $x \notin Y$ и тогда благодаря 7.3 и лемме 3.15

$$
\begin{gathered}
x \simeq P \uparrow_{X} \Rightarrow P_{x} \uparrow_{X}=P \uparrow_{X} \Rightarrow P_{x} \uparrow_{Y}=P \uparrow_{Y} \\
\Rightarrow P_{x}^{\prime} \uparrow_{Y^{\prime}}=P^{\prime} \uparrow_{Y^{\prime}} \Rightarrow P_{x}^{\prime} \uparrow_{X^{\prime}}=P^{\prime} \uparrow_{X^{\prime}} \Rightarrow x^{\prime} \simeq z,
\end{gathered}
$$

откуда согласно 10.1(d) вытекает равенство $x^{\prime}=z . \triangleright$ 
10.4. Семейство экстенсиональных $B$-систем $\left(X_{\alpha}\right)_{\alpha \in \mathrm{Ord}}$ • назовем интенсиональной иерархией или, точнее, $B$-значной интенсиональной иерархией над $X_{0}$, если

$$
\begin{array}{ll}
X_{\alpha+1}-\text { надстройка над } X_{\alpha}, & \alpha \in \text { Ord; } \\
X_{\alpha}=\bigcup_{\beta<\alpha} X_{\beta}, & \alpha \in \operatorname{Lim} \text { Ord } \bullet .
\end{array}
$$

При этом $X_{\beta} \preccurlyeq X_{\alpha}$ для любых $\beta \leqslant \alpha \in$ Ord` $^{\bullet}$ (см. лемму 7.10(e)).

10.5. Лемма. Для любой экстенсиональной $B$-системы $Y$ существует интенсиональная иерархия $\left(X_{\alpha}\right)_{\alpha \in \mathrm{Ord}}$ ' такая, что $X_{0} \leftrightarrow_{B} Y$.

$\triangleleft$ Требуемую иерархию несложно построить на основе лемм 7.10 и 10.2 с помощью конструкции индуктивного предела (см., например, [8, III.1.11]). Действительно, определим семейство $B$-систем $Y_{\alpha}\left(\alpha \in \mathrm{Ord}^{\bullet}\right)$ и изоморфных вложений $f_{\beta}^{\alpha}: Y_{\beta} \rightarrow Y_{\alpha}\left(\beta \leqslant \alpha \in \operatorname{Ord}^{\bullet}\right)$, удовлетворяющих соотношениям $f_{\alpha}^{\alpha}=\operatorname{id}_{Y_{\alpha}}$ и $f_{\gamma}^{\alpha}=f_{\beta}^{\alpha} \circ f_{\gamma}^{\beta}\left(\gamma \leqslant \beta \leqslant \alpha \in \operatorname{Ord}^{\bullet}\right)$, посредством следующей рекурсивной процедуры:

$$
\begin{aligned}
& Y_{0}:=Y, f_{0}^{0}:=\operatorname{id}_{Y_{0}} \\
& \text { для } \alpha \in \text { Ord } \\
& Y_{\alpha+1} \text { - надстройка над копией } B \text {-системы } Y_{\alpha} \text { (см. пп. 10.1, 10.2), } \\
& f_{\alpha}^{\alpha+1}-\text { соответствующий изоморфизм } Y_{\alpha} \text { на подсистему } Y_{\alpha+1} \text {, } \\
& f_{\alpha+1}^{\alpha+1}:=\operatorname{id}_{Y_{\alpha+1}}, f_{\beta}^{\alpha+1}:=f_{\alpha}^{\alpha+1} \circ f_{\beta}^{\alpha} \text { для } \beta<\alpha+1 \text {; } \\
& \text { для } \alpha \in \operatorname{Lim} \operatorname{Ord}^{\bullet} \\
& Y_{\alpha}:=\left(\bigcup_{\beta<\alpha}\{\beta\} \times Y_{\beta}\right) / \sim, \\
& \text { где для } \beta, \gamma<\alpha, \mu:=\max \{\beta, \gamma\}, x \in Y_{\beta}, y \in Y_{\gamma} \\
& (\beta, x) \sim(\gamma, y) \Leftrightarrow f_{\beta}^{\mu}(x)=f_{\gamma}^{\mu}(y), \\
& ={ }_{Y_{\alpha}}(\sim(\beta, x), \sim(\gamma, y)):==_{Y_{\mu}}\left(f_{\beta}^{\mu}(x), f_{\gamma}^{\mu}(y)\right) \text {, } \\
& \epsilon_{Y_{\alpha}}(\sim(\beta, x), \sim(\gamma, y)):=\in_{Y_{\mu}}\left(f_{\beta}^{\mu}(x), f_{\gamma}^{\mu}(y)\right) \text {, } \\
& f_{\alpha}^{\alpha}:=\operatorname{id}_{Y_{\alpha}}, f_{\beta}^{\alpha}(x):=\sim(\beta, x) \text { для } \beta<\alpha, x \in Y_{\beta} \text {. }
\end{aligned}
$$

Из 7.3, 7.10 и 10.1 следует, что семейство $B$-систем $X_{\alpha}:=f_{\alpha}^{\infty}\left(Y_{\alpha}\right)\left(\alpha \in \operatorname{Ord}^{\bullet}\right)$ является искомой интенсиональной иерархией. $\triangleright$

10.6. Лемма. Пусть $\left(X_{\alpha}\right)_{\alpha \in \mathrm{Ord}} \bullet$ и $\left(Y_{\alpha}\right)_{\alpha \in \mathrm{Ord}} \bullet-B$-значные интенсиональные иерархии.

(a) Если $f: X_{\infty} \leftrightarrow_{B} Y_{\infty}$ и $\left.f\right|_{X_{0}}: X_{0} \leftrightarrow_{B} Y_{0}$, то $\left.f\right|_{X_{\alpha}}: X_{\alpha} \leftrightarrow_{B} Y_{\alpha}$ для всех $\alpha \in$ Ord .

(b) Если $f, g: X_{\infty} \leftrightarrow_{B} Y_{\infty}$ и $\left.f\right|_{X_{0}}=\left.g\right|_{X_{0}}$, то $\left.f\right|_{X_{\alpha}}=\left.g\right|_{X_{\alpha}}$ для всех $\alpha \in$ Ord

(c) Любой изоморфизм $f_{0}: X_{0} \leftrightarrow_{B} Y_{0}$ продолжается до единственного изоморфизма $f: X_{\infty} \leftrightarrow_{B} Y_{\infty}$.

$\triangleleft$ (a), (b) При использовании индукции по $\alpha \in \mathrm{Ord}^{\bullet}$ базовый и предельный шаги тривиальны, а шаг $\alpha \mapsto \alpha+1$ легко обосновать для утверждения (а) с помощью леммы 3.15 и 10.1(b), (c), а для утверждения (b) - с помощью (a) и леммы 10.3(a).

(c) Трансфинитной рекурсией на основе леммы 10.3(b) легко построить такое семейство изоморфизмов $f_{\alpha}: X_{\alpha} \leftrightarrow_{B} Y_{\alpha}\left(\alpha \in \mathrm{Ord}^{\bullet}\right)$, что $f_{\alpha} \subset f_{\beta}$ при $\alpha \leqslant \beta$. Тогда $f:=f_{\infty}-$ искомый изоморфизм. Единственность продолжения вытекает из (b). $\triangleright$ 
10.7. Будем говорить, что $B$-система $X$ является булевозначным $(B$-значным) универсумом над $X_{0}$, если выполняются следующие условия:

(a) $X_{0} \preccurlyeq X$;

(b) $X$ экстенсиональна;

(c) $X$ интенсиональна;

(d) элементы $X \backslash X_{0}$ предикативны;

(e) $X$ отделима над $X_{0}$;

(f) $X$ регулярна вне $X_{0}$.

Согласно следствию 6.9 из условий (b) и (c) вытекает цикличность класса $\mathscr{P}_{X}(X)$ всех предикативных элементов $X$, откуда с учетом (d) следует, что разность $X \backslash X_{0}$ является предцикличной, а значит, по теореме 9.9(b) условие (f) равносильно $\sigma$-регулярности $X$ вне $X_{0}$.

Как легко видеть, булевозначный универсум $X$ над $X_{0}$ является булевозначным универсумом над любой подсистемой $Y$, удовлетворяющей соотношениям $X_{0} \subset Y \preccurlyeq X$.

10.8. Теорема. (а) Если $\left(X_{\alpha}\right)_{\alpha \in \mathrm{Ord}}$ - интенсиональная иерархия, то $X_{\infty}-$ булевозначный универсум над $X_{0}$.

(b) Если $X-$-булевозначный универсум над $X_{0}$, то существует единственная интенсиональная иерархия $\left(X_{\alpha}\right)_{\alpha \in \mathrm{Ord}} \bullet$ такая, что $X=X_{\infty}$. При этом

$$
\begin{array}{ll}
X_{\alpha+1}=X_{\alpha} \cup \mathscr{P}_{X}\left(X_{\alpha}\right), & \alpha \in \text { Ord; } \\
X_{\alpha}=\bigcup_{\alpha<\beta} X_{\beta}, & \alpha \in \text { Lim Ord } .
\end{array}
$$

$\triangleleft$ (a) Проверим условия 10.7(a)-(f) для $X=X_{\infty}$.

$10.7(\mathrm{a})$. В силу леммы 7.10 (е) система $X_{0}$, как и каждая из систем $X_{\alpha}$, является транзитивной подсистемой $X_{\infty}$. Отметим, что по этой причине выполнение соотношения $x \simeq P \uparrow$ в любой из систем $X_{\alpha}$ равносильно его выполнению в $X_{\infty}$ (см. п. 7.3).

Условие 10.7(b) входит в определение интенсиональной иерархии.

10.7(c). Пусть $P-$ подмножество $\% X_{\infty}=\bigcup_{\alpha \in \text { Ord }}{ }^{\%} X_{\alpha}$. Выбирая для каждого элемента $p \in P$ ординал $\alpha(p)$, удовлетворяющий условию $p \in X_{\alpha(p)}$, заключаем, что $P \subset{ }^{\%} X_{\alpha}$, где $\alpha:=\vee\{\alpha(p): p \in P\}$, а значит, $P \uparrow \simeq x$ для некоторого $x \in X_{\alpha+1}$ согласно 10.1(b).

$10.7(\mathrm{~d})$. Если $x \in X_{\infty} \backslash X_{0}$, то $x \in X_{\alpha+1}$ для некоторого $\alpha \in$ Ord и поэтому $\left(\exists P \subset \% X_{\alpha}\right)(x \simeq P \uparrow)$ согласно 10.1(c).

10.7(e). Пусть $x, y \in X_{\infty}, x \simeq y$ и $x \neq y$. Положим $\alpha:=\min \{\beta \in$ Ord : $\left.x, y \in X_{\beta}\right\}$ и покажем, что $\alpha=0$. Действительно, ординал $\alpha$ не может быть предельным, поскольку в этом случае $X_{\alpha}=\bigcup_{\beta<\alpha} X_{\beta}$ и тогда имеется ординал $\beta<\alpha$, для которого $x, y \in X_{\beta}$. Если же $\alpha=\beta+1$, то $x, y \in X_{\beta}$ в силу 10.1(d).

$10.7(\mathrm{f})$. Докажем, что все системы $X_{\alpha}$ (включая $X_{\infty}$ ) регулярны вне $X_{0}$, применив индукцию по $\alpha \in$ Ord ${ }^{\bullet}$.

Случай $\alpha=0$ тривиален: система $X_{0}$, очевидно, регулярна вне $X_{0}$.

Пусть система $X_{\alpha}$ регулярна вне $X_{0}$. Согласно лемме 9.5 для доказательства регулярности $X_{\alpha+1}$ вне $X_{0}$ достаточно показать регулярность $X_{\alpha+1}$ вне $X_{\alpha}$. Из 10.1(c) с учетом леммы $3.17(\mathrm{~b})$ следует, что

$$
X_{\alpha+1} \vDash(\forall y)\left(y \in X_{\alpha} \uparrow \vee y \subset X_{\alpha} \uparrow\right) .
$$


Рассмотрим произвольный булевозначный класс $\Phi \Subset X_{\alpha+1}$ и установим истинность

$$
X_{\alpha+1} \vDash\left(\Phi \cap X_{\alpha} \uparrow=\varnothing \wedge \Phi \neq \varnothing \Rightarrow(\exists y \in \Phi)(y \cap \Phi=\varnothing)\right)
$$

путем «рассуждений внутри $X_{\alpha+1} »$ (см. п. 3.10). Пусть $\Phi \cap X_{\alpha} \uparrow=\varnothing$ и $\Phi \neq \varnothing$. Возьмем любой элемент $y \in \Phi$ и покажем, что $y \cap \Phi=\varnothing$. Действительно, из $\Phi \cap X_{\alpha} \uparrow=\varnothing$ следует $y \notin X_{\alpha} \uparrow$, а значит, $y \subset X_{\alpha} \uparrow$ согласно (24) и поэтому $y \cap \Phi \subset X_{\alpha} \uparrow \cap \Phi=\varnothing$.

Пусть теперь $\alpha \in \operatorname{Lim} \operatorname{Ord}{ }^{\bullet}$, и пусть системы $X_{\beta}$ регулярны вне $X_{0}$ для всех $\beta<\alpha$. Согласно лемме 8.4 для доказательства регулярности $X_{\alpha}$ вне $X_{0}$ достаточно рассмотреть булевозначный класс $\Phi \Subset X_{\alpha}$, удовлетворяющий неравенству $\Phi \leqslant \neg\left(X_{0} \uparrow_{X_{\alpha}}\right)$, и показать $X_{\alpha} \vDash \mu(\Phi)$ или, что равносильно,

$$
\left(\forall x \in X_{\alpha}\right) X_{\alpha} \vDash(x \in \Phi \Rightarrow(\exists y \in \Phi)(y \cap \Phi=\varnothing)) .
$$

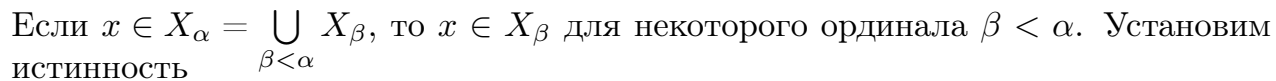

$$
X_{\alpha} \vDash(x \in \Phi \Rightarrow(\exists y \in \Phi)(y \cap \Phi=\varnothing))
$$

путем «рассуждений внутри $X_{\alpha}$. Опираясь на соотношения $\Phi \cap X_{0} \uparrow=\varnothing$, $x \in X_{\beta} \uparrow$ и $x \in \Phi$, покажем, что $(\exists y \in \Phi)(y \cap \Phi=\varnothing)$. Поскольку класс $X_{\beta} \uparrow \backslash X_{0} \uparrow$ регулярен (см. лемму 9.4) и $\varnothing \neq \Phi \cap X_{\beta} \uparrow \subset X_{\beta} \uparrow \backslash X_{0} \uparrow$, имеется такой элемент $y \in \Phi \cap X_{\beta} \uparrow$, что $y \cap \Phi \cap X_{\beta} \uparrow=\varnothing$. В силу транзитивности $X_{\beta} \uparrow$ из $y \in X_{\beta} \uparrow$ следует $y \subset X_{\beta} \uparrow$. Таким образом, $y \cap \Phi=y \cap \Phi \cap X_{\beta} \uparrow=\varnothing$.

(b) Рассмотрим семейство подсистем $X_{\alpha} \subset X$ ( $\alpha \in$ Ord $\left.{ }^{\bullet}\right)$, построенное с помощью трансфинитной рекурсии согласно (23), начиная с данной подсистемы $X_{0} \subset X$.

Индукцией по $\alpha \in \operatorname{Ord}^{\bullet}$ покажем, что $X_{\alpha} \preccurlyeq X$. Случай $\alpha=0$ содержится в условии $10.7(\mathrm{a})$. Предположим, что $X_{\alpha} \preccurlyeq X$, рассмотрим произвольные элементы $x \in X, y \in X_{\alpha+1}$ и установим неравенство $[x \in y] \leqslant\left[x \in X_{\alpha+1} \uparrow\right]$. Если $y \in X_{\alpha}$, то $[x \in y] \leqslant\left[x \in X_{\alpha} \uparrow\right] \leqslant\left[x \in X_{\alpha+1} \uparrow\right]$, а если $y \in \mathscr{P}_{X}\left(X_{\alpha}\right)$, то $y \simeq P \uparrow, P \subset{ }^{\%} X_{\alpha}$, и тогда с учетом леммы 3.17(b) имеем $[x \in y]=[x \in P \uparrow] \leqslant$ $\left[x \in X_{\alpha} \uparrow\right] \leqslant\left[x \in X_{\alpha+1} \uparrow\right]$. Если же $\alpha \in \operatorname{Lim} O r d^{\bullet}$ и $X_{\beta} \preccurlyeq X$ для всех $\beta<\alpha$, то $X_{\alpha}=\bigcup_{\alpha<\beta} X_{\beta} \preccurlyeq X$ в силу леммы 7.10(c).

Согласно лемме 7.10(а) из доказанного выше следует $X_{\alpha} \preccurlyeq X_{\alpha+1}$ для всех $\alpha \in$ Ord, что соответствует условию 10.1(а) в определении надстройки. Условия 10.1(b),(c) обеспечиваются определением $X_{\alpha+1}$, a 10.1(d) вытекает из 10.7(e). Таким образом, семейство $\left(X_{\alpha}\right)_{\alpha \in \text { Ord }}$ является интенсиональной иерархией.

Для доказательства равенства $X=X_{\infty}$ потребуется несколько вспомогательных фактов. Покажем, что

$$
(\forall x \in X)\left(\exists y \in X_{\infty}\right)[x=y]=\left[x \subset X_{\infty} \uparrow\right] .
$$

В случае $x \in X_{0}$ утверждение (25) очевидно. Пусть $x \notin X_{0}$. В силу $10.7(\mathrm{~d})$ элемент $x \in X$ предикативен, а значит, по лемме 4.10 имеется такое множество $P \subset{ }^{*} X_{\infty}$, что $\left[x \subset X_{\infty} \uparrow\right]=[x=P \uparrow]$. Согласно (а) система $X_{\infty}$ удовлетворяет условию $10.7(\mathrm{c})$, поэтому булевозначный класс $P \uparrow$ реализуется некоторым элементом $y \in X_{\infty}$, который является искомым. 
Покажем также, что

$$
(\forall x \in X)\left[x \in X_{\infty} \uparrow\right]=\left[x \subset X_{\infty} \uparrow\right]
$$

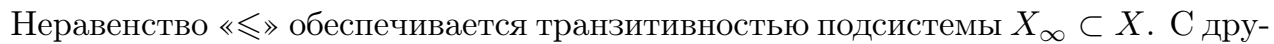
гой стороны, согласно (25) для всякого $x \in X$ имеется элемент $y \in X_{\infty}$, удовлетворяющий равенству $[x=y]=\left[x \subset X_{\infty} \uparrow\right]$, и, следовательно,

$$
\left[x \subset X_{\infty} \uparrow\right]=[x=y] \wedge\left[y \in X_{\infty} \uparrow\right] \leqslant\left[x \in X_{\infty} \uparrow\right]
$$

Переходя к доказательству равенства $X=X_{\infty}$ от противного, допустим, что существует элемент $x \in X$, не принадлежащий $X_{\infty}$. Положим $b:=\left[x \notin X_{\infty} \uparrow\right]=$ $\left[x \not \subset X_{\infty} \uparrow\right]$ (см. (26)). Заметим, что $b \neq 0_{B}$. Действительно, если $b=0_{B}$, то $X \vDash\left(x \subset X_{\infty} \uparrow\right)$ и тогда благодаря (25) найдется такой элемент $y \in X_{\infty}$, что $[x=y]=\left[x \subset X_{\infty} \uparrow\right]=1_{B}$, т. е. $x \simeq y$, откуда с учетом 10.7 (е) следует $x=y$ и тем самым $x \in X_{\infty}$. Пусть $\Phi:=\neg\left(X_{\infty} \uparrow\right)$ - дополнение $X_{\infty} \uparrow$ в $X$. Поскольку система $X$ регулярна вне $X_{0}$ (см. п. $\left.10.7(\mathrm{f})\right)$, из $\Phi \leqslant \neg\left(X_{0} \uparrow\right)$ и $[\Phi \neq \varnothing] \geqslant b$ следует $[(\exists y \in \Phi)(y \cap \Phi=\varnothing)] \geqslant b$, поэтому $[y \in \Phi \wedge y \cap \Phi=\varnothing] \neq 0_{B}$ для некоторого элемента $y \in X$. С другой стороны, согласно (26)

$$
[y \in \Phi \wedge y \cap \Phi=\varnothing]=\left[y \notin X_{\infty} \uparrow \wedge y \subset X_{\infty} \uparrow\right]=0_{B} .
$$

Единственность упомянутой в (b) интенсиональной иерархии вытекает из леммы 10.6(a). Действительно, если $\left(X_{\alpha}\right)_{\alpha \in \mathrm{Ord}} \bullet$ и $\left(Y_{\alpha}\right)_{\alpha \in \mathrm{Ord}} \bullet-$ такие интенсиональные иерархии, что $X_{0}=Y_{0}$ и $X_{\infty}=Y_{\infty}$, то для всех $\alpha \in \operatorname{Ord}^{\bullet}$ справедливо соотношение $\left.\left(\operatorname{id}_{X_{\infty}}\right)\right|_{X_{\alpha}}: X_{\alpha} \leftrightarrow_{B} Y_{\alpha}$, т. е. $X_{\alpha}=Y_{\alpha}$.

10.9. Приведенное ниже утверждение вытекает из лемм 10.5, 10.6(c) и теоремы 10.8 .

Tеорема. (а) Для любой экстенсиональной $B$-системы $Y$ существует единственный с точностью до изоморфизма $B$-значный универсум $X$ над $X_{0}$ такой, что $X_{0} \leftrightarrow_{B} Y$.

(b) Если $X$ и $Y-B$-значные универсумы над $X_{0}$ и $Y_{0}$ соответственно, то любой изоморфизм $f_{0}: X_{0} \leftrightarrow_{B} Y_{0}$ продолжается до единственного изоморфизма $f: X \leftrightarrow_{B} Y$.

Применяя утверждение (а) к какой-либо экстенсиональной $B$-системе $Y$ и рассматривая $B$-значный универсум $X$ над копией $X_{0}$ системы $Y$, условимся отождествлять $X_{0}$ с $Y$ и называть $X$ булевозначным универсумом над $Y$.

\section{$\S 11$. Булевозначный универсум}

В данном параграфе формулируются определяющие свойства классического булевозначного универсума $\mathbb{V}^{(B)}$ как алгебраической системы, доказывается существование такой системы и ее единственность с точностью до единственного изоморфизма. Эти известные факты (см. [4,9]) воспроизводятся здесь как прямые следствия установленных в $\S 10$ общих свойств булевозначного универсума над произвольной экстенсиональной системой. $\mathrm{K}$ новым результатам можно отнести приведенные ниже примеры булевозначных систем с необычными сочетаниями свойств. Эти примеры показывают, что для любой полной булевой алгебры $B$ ни одно из условий, перечисленных в определении $B$-значного универсума, не вытекает из остальных. 
11.1. Булевозначную систему $X$ называют булевозначным (точнее, $B$-значным) универсумом (см. [9, 3.4]), если она удовлетворяет следующим условиям:

(a) $X$ экстенсиональна: $X \vDash(\forall x, y)((\forall z)(z \in x \Leftrightarrow z \in y) \Rightarrow x=y)$;

(b) $X$ интенсиональна: $\left(\forall P \subset{ }^{\%} X\right)(\exists x \in X)(x \simeq P \uparrow)$;

(c) $X$ предикативна: $\quad(\forall x \in X)(\exists P \subset \% X)(x \simeq P \uparrow)$;

(d) $X$ отделима: $\quad(\forall x, y \in X)(X \vDash(x=y) \Rightarrow x=y)$;

(е) $X$ регулярна: $\quad X \vDash(\forall \Phi)((\exists y)(y \in \Phi) \Rightarrow(\exists y \in \Phi)(\forall z \in \Phi)(z \notin y))$.

Отметим, что в силу (b) и (c) регулярность (е) системы $X$ равносильна истинности в $X$ аксиомы регулярности (см. п. 9.1).

11.2. Лемма. Пусть $Y$ - произвольная экстенсиональная $B$-система. Если $B$-система $X$ является булевозначным универсумом над $Y$, то

(a) $X$ экстенсиональна;

(b) $X$ интенсиональна;

(c) $X$ предикативна тогда и только тогда, когда $Y$ предикативна;

(d) $X$ отделима тогда и только тогда, когда $Y$ отделима;

(e) $X$ регулярна тогда и только тогда, когда $Y$ регулярна.

$\triangleleft$ Утверждения (a) и (b) явно входят в определение 10.7; (c) следует из 7.3(a) и 10.7(a); (d) обеспечивается условием 10.7(e); (е) вытекает из леммы 9.7, следствия 9.6 и условия $10.7(\mathrm{f})$. $\triangleright$

11.3. Из леммы 11.2 следует, что понятие $B$-значного универсума совпадает с понятием булевозначного универсума над предикативной отделимой регулярной $B$-системой. Простейшей из таких $B$-систем является одноэлементная регулярная $B$-система. (Было бы еще проще говорить об универсуме над $\varnothing$, но алгебраическая система не может быть пустой.)

Следствие. Следующие свойства $B$-системы $X$ эквивалентны:

(a) $X$ является булевозначным универсумом;

(b) $X$ является булевозначным универсумом над $\{y\}$, где $y$ - такой элемент $X$, что $X \vDash(y=\varnothing)$;

(c) $X$ является булевозначным универсумом над одноэлементной $B$-системой $Y=\{y\}$, удовлетворяющей соотношению $Y \vDash(y \notin y)$.

11.4. Следующее утверждение, приведенное (без доказательства) в [9, 3.4], вытекает из теоремы 10.9 и следствия 11.3.

Теорема $^{1)}$. (а) Для любой полной булевой алгебры $B$ существует единственный с точностью до изоморфизма $B$-значный универсум.

(b) Для любых $B$-значных универсумов $X$ и $Y$ существует единственный изоморфизм $f: X \leftrightarrow_{B} Y$.

11.5. Булевозначный универсум, характеризуемый с точностью до изоморфизма, обозначают символом $\mathbb{V}^{(B)}$, а соответствующие оценки истинности $[\varphi]_{\mathbb{V}(B)}$ записывают в виде $\llbracket \varphi \rrbracket($ см. $[4,9])$. При этом булевозначный универсум является моделью ZFC (см. $[4,4.4])$. Точнее говоря, имеются такие определяемые с параметром $B$ классы $\mathbb{V}^{(B)}, \mathbb{\|} \cdot=\cdot \|$ и $\|\cdot \in \cdot\|$, что

$\mathrm{ZFC}, B-$ полная булева алгебра $\vdash$

$$
\begin{aligned}
& \left(\mathbb{V}^{(B)}, \mathbb{\|} \cdot=\cdot \mathbb{\|}, \mathbb{\|} \cdot \in \cdot \|\right)-B \text {-значный универсум, } \\
& \left(\mathbb{V}^{(B)}, \mathbb{\bullet} \cdot=\cdot\|,\| \cdot \in \cdot \|\right) \vDash \mathrm{ZFC} .
\end{aligned}
$$

\footnotetext{
1) Автор признателен профессору Роберту М. Соловею за плодотворное обсуждение схемы доказательства этой теоремы.
} 
Приведенные ниже примеры 11.6-11.10 показывают, что для любой полной булевой алгебры $B$ каждое из пяти условий (a)-(e), перечисленных в определении 11.1 булевозначного универсума, существенно, т. е. не вытекает из четырех остальных. Основными инструментами здесь служат теорема 10.9(а) и лемма 11.2.

11.6. ПримеР. Для любой полной булевой алгебры $B$ существует $B$-значная система, которая является интенсиональной, предикативной, отделимой и регулярной, но не экстенсиональной.

$\triangleleft$ Расширим ZFC определениями констант $2_{1}:=\{\{\varnothing\}\}$ и $2_{2}:=\{\varnothing,\{\varnothing\}\}$. Рассмотрим булевозначный универсум $\mathbb{V}^{(B)}$ и элементы $\varnothing^{\wedge},\{\varnothing\}^{\wedge}, 2_{1}^{\wedge}, 2_{2}^{\wedge} \in \mathbb{V}^{(B)}$, реализующие подъемы $\varnothing \uparrow,\left\{\varnothing^{\wedge}\right\} \uparrow,\left\{\{\varnothing\}^{\wedge}\right\} \uparrow$ и $\left\{\varnothing^{\wedge},\{\varnothing\}^{\wedge}\right\} \uparrow$ соответственно. Как легко видеть, $\mathbb{V}^{(B)} \vDash\left(2_{1}^{\wedge}=2_{1}\right)$ и $\mathbb{V}^{(B)} \vDash\left(2_{2}^{\wedge}=2_{2}\right)$ (см. п. 2.5).

Покажем, что подсистема

$$
X:=\left\{x \in \mathbb{V}^{(B)}: \mathbb{V}^{(B)} \vDash(x \not \subset\{\varnothing\})\right\} \subset \mathbb{V}^{(B)}
$$

обладает заявленными свойствами.

Согласно 2.3(а) из тавтологий $2_{1}, 2_{2} \not \subset\{\varnothing\}$ и $2_{1} \neq 2_{2}$ следует, что $2_{1}^{\wedge}, 2_{2}^{\wedge} \in X$ и $\left[2_{1}^{\wedge}=2_{2}^{\wedge}\right]_{X}=\llbracket 2_{1}^{\wedge}=2_{2}^{\wedge} \rrbracket=0_{B}$. С другой стороны, для всех $x \in X$

$$
\begin{aligned}
{\left[x \in 2_{1}^{\wedge}\right]_{X} } & =\llbracket x \in 2_{1}^{\wedge} \rrbracket=\llbracket x \in 2_{1} \wedge x \not \subset\{\varnothing\} \rrbracket=0_{B} \\
& =\llbracket x \in 2_{2} \wedge x \not \subset\{\varnothing\} \rrbracket=\llbracket x \in 2_{2}^{\wedge} \rrbracket=\left[x \in 2_{2}^{\wedge}\right]_{X} .
\end{aligned}
$$

Таким образом, $X \vDash\left(2_{1}^{\wedge} \neq 2_{2}^{\wedge}\right)$ и $X \vDash(\forall x)\left(x \in 2_{1}^{\wedge} \Leftrightarrow x \in 2_{2}^{\wedge}\right)$, а значит, система $X$ не является экстенсиональной.

Чтобы доказать интенсиональность $X$, для произвольного подмножества $P \subset{ }^{\%} X$ рассмотрим элемент $u \in \mathbb{V}^{(B)}$, реализующий подъем $P \uparrow$, положим $x:=$ $\left.\left.u\right|_{b} \sqcup 2_{1}^{\wedge}\right|_{\neg b}$ (см. п. 5.3), где $b:=\bigvee_{p \in P} \operatorname{dom} p$, и покажем, что $x \in X$ и $x \simeq P \uparrow_{X}$. Согласно лемме 3.18(a) справедливы соотношения

$$
\begin{gathered}
\llbracket P \uparrow \not \subset\{\varnothing\} \rrbracket=\llbracket(\exists y \in P \uparrow)(y \notin\{\varnothing\}) \rrbracket \\
=\bigvee_{p \in P}\left\|p \notin\{\varnothing\} \rrbracket \geqslant \bigvee_{p \in P}\right\| p \not \subset\{\varnothing\} \rrbracket=\bigvee_{p \in P} \operatorname{dom} p=b,
\end{gathered}
$$

откуда следует, что

$$
\|x \not \subset\{\varnothing\}\|=(\|u \not \subset\{\varnothing\}\| \wedge b) \vee\left(\left\|2_{1} \not \subset\{\varnothing\}\right\| \wedge \neg b\right)=(\|P \uparrow \not \subset\{\varnothing\}\| \wedge b) \vee \neg b=1_{B},
$$

т. е. $x \in X$. Кроме того, для всех $y \in X$ с учетом равенства $\llbracket y \in 2_{1}^{\wedge} \rrbracket=0_{B}$ (см. (27)) имеем

$$
\begin{aligned}
{[y \in x]_{X} } & =\| y \in x \rrbracket=(\llbracket y \in u \rrbracket \wedge b) \vee\left(\llbracket y \in 2_{1}^{\wedge} \rrbracket \wedge \neg b\right)=\llbracket y \in P \uparrow \rrbracket \wedge b \\
& =\|y \in P \uparrow \wedge P \uparrow \neq \varnothing \rrbracket=\| y \in P \uparrow \rrbracket=P \uparrow(y)=P \uparrow_{X}(y) .
\end{aligned}
$$

Предикативность и отделимость $X$ непосредственно вытекают из предикативности и отделимости $\mathbb{V}^{(B)}$, поскольку $x \Downarrow_{X} \subset x \Downarrow_{\mathbb{V}^{(B)}}$ и $[x=y]_{X}=\llbracket x=y \rrbracket$ для всех $x, y \in X$. Регулярность $X$ следует из регулярности $\mathbb{V}^{(B)}$ согласно лемме 9.7. $\triangleright$

11.7. ПримеР. Для любой полной булевой алгебры $B$ существует $B$-значная система, которая является экстенсиональной, предикативной, отделимой и регулярной, но не интенсиональной.

$\triangleleft$ Экстенсиональность, предикативность, отделимость и регулярность одноэлементной системы $\{x\}$ с интерпретациями $[x=x]=1_{B}$ и $[x \in x]=0_{B}$ очевидны. Поскольку $[x \in x]=0_{B} \neq 1_{B}=\{x\} \uparrow(x)$, булевозначный класс $\{x\} \uparrow$ не реализуется (единственным) элементом $x$, а значит, рассматриваемая система не интенсиональна. $\triangleright$ 
11.8. ПримеР. Для любой полной булевой алгебры $B$ существует $B$-значная система, которая является экстенсиональной, интенсиональной, отделимой и регулярной, но не предикативной.

$\triangleleft$ Пусть $Z$ - экстенсиональная, отделимая, регулярная $B$-значная система, носитель которой является собственным классом, отличным от класса всех множеств. (В качестве $Z$ можно взять, например, изоморфную копию $\{\varnothing\} \times \mathbb{V}^{(B)}$ булевозначного универсума $\mathbb{V}^{(B)}$.) Рассмотрим произвольное множество $\infty$, не принадлежащее $Z$, положим $Y:=Z \cup\{\infty\}$ и распространим на $Y$ булевозначные интерпретации $Z$, полагая $[\infty=\infty]_{Y}=1_{B},[\infty \in \infty]_{Y}=[z=\infty]_{Y}=[\infty=z]_{Y}=$ $[\infty \in z]_{Y}=0_{B}$ и $[z \in \infty]_{Y}=1_{B}$ для всех $z \in Z$. Элементарная проверка показывает, что $Y$ является $B$-системой.

Чтобы установить экстенсиональность $Y$, рассмотрим произвольные элементы $x, y \in Y$ и покажем, что $[(\forall z)(z \in x \Leftrightarrow z \in y)]_{Y} \leqslant[x=y]_{Y}$. Случай $x, y \in Z$ сводится к экстенсиональности $Z$, случай $x=y=\infty$ тривиален, а в случае $x \in Z$ и $y=\infty$ требуемое неравенство обеспечивается регулярностью $Z$, так как из истинности $Z \vDash \mu(\{x\} \uparrow)$ следует $Z \vDash(x \notin x)$ и поэтому

$$
[(\forall z)(z \in x \Leftrightarrow z \in \infty)]_{Y} \leqslant[x \in x \Leftrightarrow x \in \infty]_{Y}=[x \in x]_{Z}=0_{B}=[x=\infty]_{Y} .
$$

Как легко видеть, система $Y$ отделима и $Z \preccurlyeq Y$. Поскольку

$$
Y \vDash(\forall \Phi)(\Phi \cap Z \uparrow=\varnothing \Rightarrow \Phi \subset\{\infty\}),
$$

система $Y$ регулярна вне $Z$. По следствию 9.6 регулярность $Z$ влечет регулярность $Y$. Система $Y$ не является предикативной, так как насыщенный спуск $\infty \Downarrow$ содержит собственный класс $\infty \downarrow=Z$.

Из леммы 11.2 следует, что булевозначный универсум над $Y$ удовлетворяет всем требованиям, указанным в условии. $\triangleright$

11.9. Пример. Для любой полной булевой алгебры $B$ существует $B$-значная система, которая является экстенсиональной, интенсиональной, предикативной и регулярной, но не отделимой.

$\triangleleft$ Согласно лемме 11.2 всеми перечисленными свойствами обладает булевозначный универсум над двухэлементной $B$-системой $Y$ с интерпретациями $=_{Y}: Y^{2} \rightarrow\left\{1_{B}\right\}, \in_{Y}: Y^{2} \rightarrow\left\{0_{B}\right\} . \triangleright$

11.10. Пример. Для любой полной булевой алгебры $B$ существует $B$-значная система, которая является экстенсиональной, интенсиональной, предикативной и отделимой, но не регулярной.

$\triangleleft$ Поскольку одноэлементное множество $Y=\{y\}$, снабженное интерпретациями $[y=y]_{Y}=[y \in y]_{Y}=1_{B}$, является экстенсиональной, предикативной, отделимой и не регулярной $B$-системой, согласно лемме 11.2 булевозначный универсум над $Y$ обладает заявленными в условии свойствами. $\triangleright$

\section{$\S 12$. Иерархии в булевозначном универсуме}

В этом параграфе предложены описания структуры булевозначного универсума $\mathbb{V}^{(B)}$ посредством четырех иерархий, одна из которых воспроизводит интенсиональную иерархию, вторая служит спуском иерархии фон Неймана, а две другие порождаются подъемами специального вида и перемешиваниями.

В соответствии с соглашением 3.20 для всякого подмножества $P \subset \% \mathbb{V}^{(B)}$ булевозначный класс $P \uparrow \Subset \mathbb{V}^{(B)}$ отождествляется с реализующим его элементом $\mathbb{V}^{(B)}$. 
12.1. Иерархия, описанная в следующей теореме, соответствует классической конструкции (неотделимого) булевозначного универсума $[4,4.1 .2 ; 6,(14.15)]$ и за вычетом нулевого члена совпадает с интенсиональной иерархией (23) над одноэлементной регулярной $B$-системой.

Теорема. С помощью трансфинитной рекурсии определим семейство подмножеств $\mathbb{V}_{\alpha}^{(B)} \subset \mathbb{V}^{(B)}(\alpha \in$ Ord $)$, полагая

$$
\begin{array}{ll}
\mathbb{V}_{0}^{(B)}=\varnothing ; & \\
\mathbb{V}_{\alpha+1}^{(B)}=\mathscr{P}_{\mathbb{V}^{(B)}}\left(\mathbb{V}_{\alpha}^{(B)}\right), & \alpha \in \text { Ord; } \\
\mathbb{V}_{\alpha}^{(B)}=\bigcup_{\beta<\alpha} \mathbb{V}_{\beta}^{(B)}, & \alpha \in \text { Lim Ord. }
\end{array}
$$

Тогда

$$
\mathbb{V}^{(B)}=\bigcup_{\alpha \in \text { Ord }} \mathbb{V}_{\alpha}^{(B)}
$$

При этом семейство $\left(X_{\alpha}\right)_{\alpha \in \mathrm{Ord}} \bullet$, определенное по правилу

$$
X_{\alpha}= \begin{cases}\mathbb{V}_{\alpha+1}^{(B)} & \text { для } \alpha<\omega ; \\ \mathbb{V}_{\alpha}^{(B)} & \text { для } \omega \leqslant \alpha<\infty ; \\ \mathbb{V}^{(B)} & \text { для } \alpha=\infty,\end{cases}
$$

является интенсиональной иерархией над $X_{0}=\mathbb{V}_{1}^{(B)}=\{\varnothing \uparrow\}$. В частности, $\mathbb{V}_{\beta}^{(B)}-$ транзитивное подмножество $\mathbb{V}_{\alpha}^{(B)}$ для всех $\beta \leqslant \alpha \in \operatorname{Ord}{ }^{\bullet}$, где $\mathbb{V}_{\infty}^{(B)}:=\mathbb{V}^{(B)}$.

$\triangleleft$ Индукцией по $\alpha \in$ Ord покажем, что $\mathbb{V}_{\beta}^{(B)} \subset \mathbb{V}_{\alpha}^{(B)}$ при $\beta<\alpha$. Пусть $\mathbb{V}_{\gamma}^{(B)} \subset \mathbb{V}_{\beta}^{(B)}$ для всех $\gamma<\beta<\alpha$. Рассмотрим произвольные $\beta<\alpha$ и $x \in \mathbb{V}_{\beta}^{(B)}$ и покажем, что $x \in \mathbb{V}_{\alpha}^{(B)}$. Случаи $\alpha=0$ и $\alpha \in \operatorname{Lim}$ Ord тривиальны. Пусть $\alpha=\alpha_{0}+1$. Из определения (28) следует, что $x \in \mathscr{P}_{\mathrm{V}}^{(B)}\left(\mathbb{V}_{\gamma}^{(B)}\right)$ для некоторого ординала $\gamma<$ $\beta \leqslant \alpha_{0}<\alpha$. По предположению индукции $\mathbb{V}_{\gamma}^{(B)} \subset \mathbb{V}_{\alpha_{0}}^{(B)}$, а значит, $x \in \mathscr{P}_{\mathbb{V}^{(B)}}\left(\mathbb{V}_{\gamma}^{(B)}\right) \subset$ $\mathscr{P}_{\mathrm{V}}(B)\left(\mathbb{V}_{\alpha_{0}}^{(B)}\right)=\mathbb{V}_{\alpha}^{(B)}$.

Из включений $X_{\alpha} \subset X_{\alpha+1}$ следует, что $X_{\alpha+1}=X_{\alpha} \cup \mathscr{P}_{X_{\infty}}\left(X_{\alpha}\right)$ для всех $\alpha \in$ Ord. Остается воспользоваться теоремой 10.8(b). $\triangleright$

12.2. Следствие $[4,4.1 .3]$. Пусть $C$ - произвольный подкласс $\mathbb{V}^{(B)}$. Если $P \uparrow \in C$ для всех $P \subset{ }^{\%} C$, то $C=\mathbb{V}^{(B)}$.

$\triangleleft$ Допустим, $C \neq \mathbb{V}^{(B)}$. По теореме 12.1 существует наименьший ординал $\alpha$, для которого имеется элемент $x \in \mathbb{V}_{\alpha}^{(B)} \backslash C$. Из (28) видно, что $\alpha \neq 0$ и $\alpha \notin \operatorname{Lim}$ Ord. C другой стороны, если $\alpha=\beta+1$, то $x=P \uparrow$ для некоторого подмножества $P \subset \% \mathbb{V}_{\beta}^{(B)}$, и тогда из $\mathbb{V}_{\beta}^{(B)} \subset C$ вытекает $x \in C$.

12.3. Поскольку булевозначный универсум $\mathbb{V}^{(B)}$ является моделью ZFC, в нем истинно утверждение

$$
\mathbb{V}=\bigcup_{\alpha \in \text { Ord }} \mathbb{V}_{\alpha}
$$

о представимости класса всех множеств $\mathbb{V}$ в виде объединения классической кумулятивной иерархии фон Неймана $\left(\mathbb{V}_{\alpha}\right)_{\alpha \in \text { Ord }}$ (см. (15)).

Определим спуск иерархии $\left(\mathbb{V}_{\alpha}\right)_{\alpha \in \text { Ord }}$ из $\mathbb{V}^{(B)}$, сопоставляя каждому ординалу $\alpha$ спуск $\mathscr{V}_{\alpha} \downarrow$ того элемента $\mathscr{V}_{\alpha} \in \mathbb{V}^{(B)}$, который внутри $\mathbb{V}^{(B)}$ равен соответствующему члену $\mathbb{V}_{\alpha^{\wedge}}$ иерархии фон Неймана (см. [4, 4.4.10]):

$$
\mathbb{V}^{(B)} \vDash\left(\mathscr{V}_{\alpha}=\mathbb{V}_{\alpha^{\wedge}}\right) .
$$


Было бы естественно ожидать, что иерархия (28), являющаяся булевозначным аналогом иерархии (15), совпадает со спуском последней: $\left(\mathbb{V}_{\alpha}^{(B)}\right)_{\alpha \in \text { Ord }}=$ $\left(\mathscr{V}_{\alpha} \downarrow\right)_{\alpha \in \mathrm{Ord}}$. Тем не менее в случае бесконечной булевой алгебры $B$ это не так, поскольку не все подмножества $\mathbb{V}_{\alpha}^{(B)} \subset \mathbb{V}^{(B)}$ цикличны. Действительно, если $\left(d_{n}\right)_{n \in \omega}-$ разбиение единицы, составленное из ненулевых элементов $d_{n} \in B$, то соединение $\left.\bigsqcup_{n \in \omega} n^{\wedge}\right|_{d_{n}}$ принадлежит $\mathscr{V} \downarrow \downarrow$, но не принадлежит $\mathbb{V}_{\omega}^{(B)}=\bigcup_{n \in \omega} \mathbb{V}_{n}^{(B)}$.

Следующее утверждение показывает, что для превращения иерархии (28) в спуск иерархии фон Неймана достаточно добавить перемешивания на предельных шагах.

Теорема. С помощью трансфинитной рекурсии определим семейство подмножеств $\mathbb{U}_{\alpha}^{(B)} \subset \mathbb{V}^{(B)}(\alpha \in$ Ord $)$, полагая

$$
\begin{aligned}
& \mathbb{U}_{0}^{(B)}=\varnothing ; \\
& \mathbb{U}_{\alpha+1}^{(B)}=\mathscr{P}_{\mathbb{V}^{(B)}}\left(\mathbb{U}_{\alpha}^{(B)}\right), \quad \alpha \in \text { Ord } ; \\
& \mathbb{U}_{\alpha}^{(B)}=\operatorname{mix} \bigcup_{\beta<\alpha} \mathbb{U}_{\beta}^{(B)}, \quad \alpha \in \text { Lim Ord. }
\end{aligned}
$$

Тогда $\left(\mathbb{U}_{\alpha}^{(B)}\right)_{\alpha \in \text { Ord }}-$ спуск иерархии фон Неймана $\left(\mathbb{V}_{\alpha}\right)_{\alpha \in \text { Ord }}$ из $\mathbb{V}^{(B)}$, т. е.

$$
\mathbb{U}_{\alpha}^{(B)}=\mathscr{V}_{\alpha} \downarrow \text { для всех } \alpha \in \text { Ord, }
$$

где $\mathscr{V}_{\alpha}-$ элементы $\mathbb{V}^{(B)}$, удовлетворяющие соотношениям $\mathbb{V}^{(B)} \vDash\left(\mathscr{V}_{\alpha}=\mathbb{V}_{\alpha^{\wedge}}\right)$.

$\triangleleft$ Докажем равенство $\mathbb{U}_{\alpha}^{(B)}=\mathscr{V}_{\alpha} \downarrow$ индукцией по $\alpha \in$ Ord.

База индукции $\alpha=0$ тривиальна: $\mathbb{U}_{0}^{(B)}=\varnothing=\varnothing \uparrow \downarrow=\mathscr{V}_{0} \downarrow$.

Если $\mathbb{U}_{\alpha}^{(B)}=\mathscr{V}_{\alpha} \downarrow$, то, учитывая соотношение

$$
\mathbb{V}^{(B)} \vDash\left(\mathscr{P}\left(\mathbb{U}_{\alpha}^{(B) \uparrow}\right)=\mathscr{P}\left(\mathscr{V}_{\alpha} \downarrow \uparrow\right)=\mathscr{P}\left(\mathscr{V}_{\alpha}\right)=\mathscr{P}\left(\mathbb{V}_{\alpha^{\wedge}}\right)=\mathbb{V}_{\alpha^{\wedge}+1}=\mathbb{V}_{(\alpha+1)^{\wedge}}=\mathscr{V}_{\alpha+1}\right)
$$

и используя лемму 3.17 (b),(c) и следствие 6.4 , заключаем, что для всех $x \in \mathbb{V}^{(B)}$

$$
\begin{aligned}
& x \in \mathbb{U}_{\alpha+1}^{(B)} \Leftrightarrow x \in \mathscr{P}_{\mathbb{V}(B)}\left(\mathbb{U}_{\alpha}^{(B)}\right) \Leftrightarrow\left(\exists P \subset{ }^{\%} \mathbb{U}_{\alpha}^{(B)}\right)(x=P \uparrow) \Leftrightarrow \mathbb{V}^{(B)} \vDash\left(x \subset \mathbb{U}_{\alpha}^{(B) \uparrow}\right) \\
& \Leftrightarrow \mathbb{V}^{(B)} \vDash\left(x \in \mathscr{P}\left(\mathbb{U}_{\alpha}^{(B)} \uparrow\right)\right) \Leftrightarrow \mathbb{V}^{(B)} \vDash\left(x \in \mathscr{V}_{\alpha+1}\right) \Leftrightarrow x \in \mathscr{V}_{\alpha+1} \downarrow .
\end{aligned}
$$

Если $\alpha \in \operatorname{Lim}$ Ord и $\mathbb{U}_{\beta}^{(B)}=\mathscr{V}_{\beta} \downarrow$ для всех $\beta<\alpha$, то с учетом равенства $\alpha^{\wedge}=\left\{\beta^{\wedge}: \beta \in \alpha\right\} \uparrow$, леммы 3.18(a), п. 5.10 и следствия 6.4 для всех $x \in \mathbb{V}^{(B)}$ имеем

$$
\begin{gathered}
x \in \mathbb{U}_{\alpha}^{(B)} \Leftrightarrow x \in \operatorname{mix} \bigcup_{\beta<\alpha} \mathbb{U}_{\beta}^{(B)} \Leftrightarrow \vee\left\{\llbracket x=y \rrbracket: y \in \bigcup_{\beta<\alpha} \mathbb{U}_{\beta}^{(B)}\right\}=1_{B} \\
\Leftrightarrow \bigvee_{\beta<\alpha} \bigvee_{y \in \mathbb{U}_{\beta}^{(B)}} \llbracket x=y \rrbracket=1_{B} \Leftrightarrow \bigvee_{\beta<\alpha} \llbracket x \in \mathbb{U}_{\beta}^{(B)} \uparrow \rrbracket=1_{B} \Leftrightarrow \bigvee_{\beta<\alpha} \llbracket x \in \mathscr{V}_{\beta} \downarrow \uparrow \rrbracket=1_{B} \\
\Leftrightarrow \bigvee_{\beta<\alpha}\left\|x \in \mathscr{V}_{\beta} \rrbracket=1_{B} \Leftrightarrow \bigvee_{\beta \in \alpha}\right\| x \in \mathbb{V}_{\beta^{\wedge}} \rrbracket=1_{B} \Leftrightarrow \mathbb{V}^{(B)} \vDash\left(\exists \beta \in \alpha^{\wedge}\right)\left(x \in \mathbb{V}_{\beta}\right) \\
\Leftrightarrow \mathbb{V}^{(B)} \vDash\left(x \in \bigcup_{\beta<\alpha^{\wedge}} \mathbb{V}_{\beta}\right) \Leftrightarrow \mathbb{V}^{(B)} \vDash\left(x \in \mathbb{V}_{\alpha^{\wedge}}\right) \Leftrightarrow \mathbb{V}^{(B)} \vDash\left(x \in \mathscr{V}_{\alpha}\right) \Leftrightarrow x \in \mathscr{V}_{\alpha \downarrow} . \triangleright
\end{gathered}
$$

12.4. В построении иерархии (28) задействованы подъемы $P \uparrow$ произвольных множеств $P$ частичных элементов:

$$
\mathbb{V}_{\alpha+1}^{(B)}=\mathscr{P}_{\mathbb{V}(B)}\left(\mathbb{V}_{\alpha}^{(B)}\right)=\left\{P \uparrow: P \subset \mathbb{V}_{\alpha}^{(B)}\right\} .
$$


С другой стороны, согласно следствию 5.15(b) всякий элемент булевозначного универсума представляется в виде подъема $\left(\left.Y\right|_{b}\right) \uparrow$ множества $\left.Y\right|_{b}$ частичных элементов с одной и той же областью определения $b$. В этой связи выглядит естественной гипотеза о том, что булевозначный универсум может быть выстроен в иерархию подъемов вида $\left(\left.Y\right|_{b}\right) \uparrow$. Тем не менее приведенный ниже факт опровергает эту гипотезу.

Теорема. Пусть отделимая экстенсиональная $B$-система $X$ удовлетворяет принципу подъема. C помощью трансфинитной рекурсии определим семейство подмножеств $Y_{\alpha} \subset X(\alpha \in \mathrm{Ord})$, полагая

$$
Y_{\alpha}=\left\{\left(\left.Y\right|_{b}\right) \uparrow: Y \subset \bigcup_{\beta<\alpha} Y_{\beta}, b \in B\right\}, \quad \alpha \in \text { Ord. }
$$

Если булева алгебра $B$ бесконечна, то $\bigcup_{\alpha \in \mathrm{Ord}} Y_{\alpha} \neq X$.

$\triangleleft$ Рекурсивно определим последовательность $\left(y_{n}\right)_{n \in \omega} \subset X$, полагая

$$
y_{0}:=\varnothing \uparrow, \quad y_{n+1}:=\left\{y_{n}\right\} \uparrow, \quad n \in \omega .
$$

Зафиксируем произвольную антицепь $\left(d_{n}\right)_{n \in \omega}$ ненулевых элементов $B$ и рассмотрим подъемы

$$
x_{i}:=\left\{\left.y_{n}\right|_{d_{n+i}}: n \in \omega\right\} \uparrow, \quad i \in \omega .
$$

Поскольку $x_{i}=\left(\left.\bigcup_{n \in \omega}\left\{y_{n}\right\}\right|_{d_{n+i}}\right) \uparrow$ и $\left\{y_{n}\right\} \uparrow=y_{n+1}$, согласно лемме $6.8^{2)}$ справедливы соотношения

$$
x_{i}=\left.\operatorname{ext} \bigsqcup_{n \in \omega} y_{n+1}\right|_{d_{n+i}}, \quad i \in \omega .
$$

Для завершения доказательства достаточно установить, что $x_{i} \notin Y_{\alpha}$ при всех $\alpha \in \mathrm{Ord}, i \in \omega$. Воспользуемся трансфинитной индукцией по $\alpha$. Рассмотрим $\alpha \in$ Ord, предположим, что $x_{i} \notin Y_{\beta}$ для всех $\beta<\alpha, i \in \omega$, и допустим, что $x_{i} \in Y_{\alpha}$ для некоторого $i \in \omega$. Тогда $x_{i}=\left(\left.Y\right|_{b}\right) \uparrow$, где $Y \subset \bigcup_{\beta<\alpha} Y_{\beta}, b \in B$. При этом $\left[y \in x_{i}\right]=\left[x_{i} \neq \varnothing\right]=\bigvee_{n \in \omega} d_{n+i}=b$ для всех $y \in Y$ (см. следствие 5.15(b)). Поскольку $\left[x_{i} \neq \varnothing\right] \neq 0_{B}$, множество $Y$ непусто, а значит, существуют $\beta<\alpha$ и $y \in Y_{\beta}$ такие, что $\left[y \in x_{i}\right]=b$. С учетом (30) имеем

$$
\bigvee_{n \in \omega} d_{n+i}=\left[y \in x_{i}\right]=\bigvee_{n \in \omega}\left[y=y_{n}\right] \wedge d_{n+i}
$$

откуда по лемме $5.1\left(\right.$ а) следует, что $d_{n+i}=\left[y=y_{n}\right] \wedge d_{n+i}$, т. е. $\left[y=y_{n}\right] \geqslant d_{n+i}$ для всех $n \in \omega$, а значит, $\left.y\right|_{b}=\left.\bigsqcup_{n \in \omega} y_{n}\right|_{d_{n+i}}$. Учитывая равенство $y_{0}=\varnothing \uparrow$ и соот-
ношение (31), заключаем:

$$
\left.\operatorname{ext} y\right|_{b}=\left.\operatorname{ext} \bigsqcup_{n \in \omega} y_{n+1}\right|_{d_{(n+1)+i}}=\left.\operatorname{ext} \bigsqcup_{n \in \omega} y_{n+1}\right|_{d_{n+(i+1)}}=x_{i+1} \text {. }
$$
Поскольку $y \in Y_{\beta}$, имеются $Z \subset \bigcup_{\gamma<\beta} Y_{\gamma}$ и $c \in B$ такие, что $y=\left(\left.Z\right|_{c}\right) \uparrow$. Тогда для
всех $x \in X$

$$
\begin{gathered}
{\left[\left.x \in \operatorname{ext} y\right|_{b}\right]=\left[\left.x \in y\right|_{b}\right]=[x \in y] \wedge b=\left[x \in\left(\left.Z\right|_{c}\right) \uparrow\right] \wedge b} \\
=\bigvee_{z \in Z}\left[x=\left.z\right|_{c}\right] \wedge b=\bigvee_{z \in Z}\left[x=\left.z\right|_{c \wedge b}\right]=\left[x \in\left(\left.Z\right|_{c \wedge b}\right) \uparrow\right],
\end{gathered}
$$

откуда с учетом (32) следует, что $x_{i+1}=\left.\operatorname{ext} y\right|_{b}=\left(\left.Z\right|_{c \wedge b}\right) \uparrow \in Y_{\beta}$ вопреки предположению индукции.

\footnotetext{
${ }^{2)} \mathrm{B}$ формулировке леммы 6.8 в [1] имеется опечатка: вместо $\bigsqcup_{i \in I} x_{i}$ должно быть $\left.\bigsqcup_{i \in I} x_{i}\right|_{d_{i}}$.
} 
12.5. Лемма. Если $\left(Z_{i}\right)_{i \in I}-$ семейство подмножеств $\mathbb{V}^{(B)},\left(d_{i}\right)_{i \in I} \subset B-$ разбиение единицы и $\left(b_{i}\right)_{i \in I} \subset B$, то существуют такие $Y \subset \operatorname{mix} \bigcup_{i \in I} Z_{i}$ и $b \in B$, что

$$
\left.\bigsqcup_{i \in I}\left(\left.Z_{i}\right|_{b_{i}}\right) \uparrow\right|_{d_{i}}=\left(\left.Y\right|_{b}\right) \uparrow .
$$

$\triangleleft$ Положим $Z:=\bigcup_{i \in I} Z_{i} \subset \mathbb{V}^{(B)}$ и $P:=\left.\bigcup_{i \in I} Z_{i}\right|_{b_{i} \wedge d_{i}} \subset{ }^{\%} Z$. Согласно лемме 6.8 имеет место равенство $\left.\bigsqcup_{i \in I}\left(\left.Z_{i}\right|_{b_{i}}\right) \uparrow\right|_{d_{i}}=P \uparrow$, а благодаря следствию 5.15(а) существуют $Y \subset \operatorname{mix} Z$ и $b \in B$ такие, что $P \uparrow=\left(\left.Y\right|_{b}\right) \uparrow . \triangleright$

12.6. Согласно теореме 12.4 для построения булевозначного универсума недостаточно одних только подъемов с постоянной областью определения. Приведенные ниже результаты показывают, что ситуация изменится, если на предельных шагах иерархии добавить перемешивания.

Теорема. $C$ помощью трансфинитной рекурсии определим семейство подмножеств $Y_{\alpha} \subset \mathbb{V}^{(B)}(\alpha \in$ Ord $)$, полагая

$$
\begin{array}{ll}
Y_{0}=\varnothing ; & \\
Y_{\alpha+1}=\left\{\left(\left.Y\right|_{b}\right) \uparrow: Y \subset Y_{\alpha}, b \in B\right\}, & \alpha \in \text { Ord; } \\
Y_{\alpha}=\operatorname{mix} \bigcup_{\beta<\alpha} Y_{\beta}, & \alpha \in \text { Lim Ord. }
\end{array}
$$

Тогда имеют место следующие соотношения:

(a) $Y_{\alpha} \subset Y_{\beta}$ при $\alpha \leqslant \beta$;

(b) множества $Y_{\alpha}$ цикличны для всех $\alpha \in$ Ord;

(c) $\mathbb{V}_{\alpha}^{(B)} \subset Y_{\alpha+1}$ для всех $\alpha \in$ Ord;

(d) $\mathbb{V}^{(B)}=\bigcup_{\alpha \in \text { Ord }} Y_{\alpha}$

$\triangleleft$ (а) Докажем, что $Y_{\alpha} \subset Y_{\beta}$ для всех $\alpha \leqslant \beta$, воспользовавшись индукцией по $\beta$. Пусть $\beta \in$ Ord, и пусть

$$
Y_{\alpha_{1}} \subset Y_{\alpha_{2}} \text { для всех } \alpha_{1} \leqslant \alpha_{2}<\beta \text {. }
$$

Рассмотрим произвольный ординал $\alpha \leqslant \beta$ и установим включение $Y_{\alpha} \subset Y_{\beta}$. Это включение очевидно, если $\alpha=0, \alpha=\beta$ или $\beta \in \operatorname{Lim}$ Ord. Поэтому будем считать, что $0<\alpha<\beta$ и $\beta=\beta_{0}+1$ для некоторого $\beta_{0} \in$ Ord. Зафиксируем произвольный элемент $x \in Y_{\alpha}$ и покажем, что $x \in Y_{\beta}$.

Если $\alpha=\alpha_{0}+1$ для некоторого $\alpha_{0} \in$ Ord, то $x=\left(\left.Y\right|_{b}\right) \uparrow$, где $Y \subset Y_{\alpha_{0}}, b \in B$. Поскольку $\alpha_{0}<\beta_{0}<\beta$, в силу (34) справедливо включение $Y_{\alpha_{0}} \subset Y_{\beta_{0}}$, а значит, $Y \subset Y_{\beta_{0}}$ и, следовательно, $x=\left(\left.Y\right|_{b}\right) \uparrow \in Y_{\beta_{0}+1}=Y_{\beta}$.

Пусть теперь $\alpha \in \operatorname{Lim}$ Ord. Тогда $Y_{\alpha}=\operatorname{mix} \bigcup_{\gamma<\alpha} Y_{\gamma}$ и поэтому $x=\left.\bigsqcup_{i \in I} x_{i}\right|_{d_{i}}$, где $\left(d_{i}\right)_{i \in I} \subset B-$ разбиение единицы, $x_{i} \in Y_{\gamma_{i}}, \gamma_{i}<\alpha(i \in I)$. Поскольку ординал $\alpha$ является предельным, для всех $i \in I$ имеет место неравенство $\gamma_{i}+1<\alpha$ и, в частности, $\gamma_{i}<\gamma_{i}+1<\beta$, откуда благодаря (34) следует, что $Y_{\gamma_{i}} \subset Y_{\gamma_{i}+1}$. Таким образом, $x_{i} \in Y_{\gamma_{i}+1}$, а значит, $x_{i}=\left(\left.Z_{i}\right|_{b_{i}}\right) \uparrow$ для некоторых $Z_{i} \subset Y_{\gamma_{i}}$, $b_{i} \in B(i \in I)$ и тем самым $x=\left.\bigsqcup_{i \in I}\left(\left.Z_{i}\right|_{b_{i}}\right) \uparrow\right|_{d_{i}}$. Согласно лемме 12.5 существуют такие $Y \subset \operatorname{mix} \bigcup_{i \in I} Z_{i}$ и $b \in B$, что $x=\left(\left.Y\right|_{b}\right) \uparrow$. Заметим, что

$$
\operatorname{mix} \bigcup_{i \in I} Z_{i} \subset \operatorname{mix} \bigcup_{i \in I} Y_{\gamma_{i}} \subset \operatorname{mix} \bigcup_{\gamma<\alpha} Y_{\gamma}=Y_{\alpha}
$$


и, кроме того, в силу (34) из $\alpha \leqslant \beta_{0}<\beta$ вытекает $Y_{\alpha} \subset Y_{\beta_{0}}$. Следовательно, $Y \subset Y_{\beta_{0}}$ и поэтому $x=\left(\left.Y\right|_{b}\right) \uparrow \in Y_{\beta_{0}+1}=Y_{\beta}$.

(b) Применяя индукцию, рассмотрим произвольный ординал $\alpha$, предположим, что $\operatorname{mix} Y_{\beta}=Y_{\beta}$ для всех $\beta<\alpha$, и установим равенство $\operatorname{mix} Y_{\alpha}=Y_{\alpha}$. В случае $\alpha=0$ или $\alpha \in \operatorname{Lim}$ Ord это равенство очевидно. Пусть $\alpha=\beta+1$ для некоторого $\beta \in$ Ord. Рассмотрим произвольный элемент $x \in \operatorname{mix} Y_{\alpha}$ и покажем, что $x \in Y_{\alpha}$. Поскольку $x \in \operatorname{mix} Y_{\beta+1}=\operatorname{mix}\left\{\left(\left.Z\right|_{b}\right) \uparrow: Z \subset Y_{\beta}, b \in B\right\}$, имеются разбиение единицы $\left(d_{i}\right)_{i \in I} \subset B$ и семейства $Z_{i} \subset Y_{\beta}, b_{i} \in B(i \in I)$ такие, что $x=\left.\bigsqcup_{i \in I}\left(\left.Z_{i}\right|_{b_{i}}\right) \uparrow\right|_{d_{i}}$. Из леммы 12.5 вытекает представление $x=\left(\left.Y\right|_{b}\right) \uparrow$ для некоторых $Y \subset \operatorname{mix} \bigcup_{i \in I} Z_{i}$ и $b \in B$. По предположению индукции имеем $\operatorname{mix} \bigcup_{i \in I} Z_{i} \subset \operatorname{mix} Y_{\beta}=Y_{\beta}$, а значит, $Y \subset Y_{\beta}$ и тем самым $x=\left(\left.Y\right|_{b}\right) \uparrow \in Y_{\beta+1}=Y_{\alpha}$.

(c) Вновь действуя по индукции, зафиксируем ординал $\alpha$, предположим, что $\mathbb{V}_{\beta}^{(B)} \subset Y_{\beta+1}$ для всех $\beta<\alpha$, рассмотрим произвольный элемент $x \in \mathbb{V}_{\alpha}^{(B)}$ и покажем, что $x \in Y_{\alpha+1}$. Из (28) следует, что $x=P \uparrow$ для некоторого подмножества $P \subset{ }^{*} \mathbb{V}_{\beta}^{(B)}$, где $\beta<\alpha$. Согласно следствию 5.15(а) имеются $Y \subset \operatorname{mix} \mathbb{V}_{\beta}^{(B)}$ и $b \in B$ такие, что $x=\left(\left.Y\right|_{b}\right) \uparrow$. По предположению индукции $\mathbb{V}_{\beta}^{(B)} \subset Y_{\beta+1}$. Кроме того, из (а) вытекает включение $Y_{\beta+1} \subset Y_{\alpha}$. Таким образом, $\mathbb{V}_{\beta}^{(B)} \subset Y_{\alpha}$, откуда с учетом (b) следует $Y \subset \operatorname{mix} \mathbb{V}_{\beta}^{(B)} \subset \operatorname{mix} Y_{\alpha}=Y_{\alpha}$ и поэтому $x=\left(\left.Y\right|_{b}\right) \uparrow \in Y_{\alpha+1}$.

Равенство (d) вытекает из (c) и (29).

12.7. Следующее утверждение показывает, что равенство 12.6(d) сохранит силу, если на дискретных шагах иерархии (33) ограничиться подъемами множеств всюду определенных элементов.

Теорема. С помощью трансфинитной рекурсии определим семейство подмножеств $Z_{\alpha} \subset \mathbb{V}^{(B)}(\alpha \in$ Ord), полагая

$$
\begin{array}{ll}
Z_{0}=\varnothing ; & \\
Z_{\alpha+1}=\left\{Z \uparrow: Z \subset Z_{\alpha}\right\}, & \alpha \in \text { Ord; } \\
Z_{\alpha}=\operatorname{mix} \bigcup_{\beta<\alpha} Z_{\beta}, & \alpha \in \text { Lim Ord }
\end{array}
$$

Тогда

$$
\mathbb{V}^{(B)}=\bigcup_{\alpha \in \text { Ord }} Z_{\alpha}
$$

$\triangleleft$ Определим функцию $\delta:$ Ord $\rightarrow$ Ord следующей рекурсивной формулой:

$$
\delta(\alpha)=\vee\{\delta(\beta): \beta<\alpha\}+\omega, \quad \alpha \in \text { Ord }
$$

Благодаря теореме 12.6(d) достаточно доказать, что

$$
(\forall \alpha \in \operatorname{Ord})\left(Y_{\alpha} \subset Z_{\delta(\alpha)}\right),
$$

где $Y_{\alpha}$ определены в (33). Используя индукцию по $\alpha \in$ Ord, предположим, что $Y_{\beta} \subset Z_{\delta(\beta)}$ для всех $\beta<\alpha$, и установим включение $Y_{\alpha} \subset Z_{\delta(\alpha)}$.

Пусть $\alpha=\beta+1$. Из очевидной монотонности функции $\delta$ следует, что

$$
\delta(\alpha)=\vee\{\delta(\gamma): \gamma<\alpha\}+\omega=\vee\{\delta(\gamma): \gamma \leqslant \beta\}+\omega=\delta(\beta)+\omega .
$$


Рассмотрим произвольный элемент $x \in Y_{\alpha}=Y_{\beta+1}$. Согласно (33) имеются $Y \subset Y_{\beta}$ и $b \in B$ такие, что $x=\left(\left.Y\right|_{b}\right) \uparrow$. Тогда для всех $z \in \mathbb{V}^{(B)}$

$$
\begin{gathered}
\llbracket z \in x \rrbracket=\llbracket z \in\left(\left.Y\right|_{b}\right) \uparrow \rrbracket=\bigvee_{y \in Y} \llbracket z=\left.y\right|_{b} \rrbracket=\bigvee_{y \in Y} \llbracket z=y \rrbracket \wedge b=\llbracket z \in Y \uparrow \rrbracket \wedge b \\
=\left.\llbracket z \in(Y \uparrow)\right|_{b} \rrbracket=\left.\llbracket z \in(Y \uparrow)\right|_{b} \rrbracket \vee 0_{B}=\left.\left.\llbracket z \in(Y \uparrow)\right|_{b} \rrbracket \vee \llbracket z \in(\varnothing \uparrow)\right|_{\neg b} \rrbracket,
\end{gathered}
$$

откуда по лемме 5.6(d) вытекает равенство $x=\left.\left.(Y \uparrow)\right|_{b} \sqcup \varnothing \uparrow\right|_{\neg b}$. Из включений $Y, \varnothing \subset Y_{\beta} \subset Z_{\delta(\beta)}$ следует $Y \uparrow, \varnothing \uparrow \in Z_{\delta(\beta)+1}$ и поэтому

$$
x=\left.\left.(Y \uparrow)\right|_{b} \sqcup \varnothing \uparrow\right|_{\neg b} \in \operatorname{mix} Z_{\delta(\beta)+1} \subset \operatorname{mix} \bigcup_{\gamma<\delta(\beta)+\omega} Z_{\gamma}=Z_{\delta(\beta)+\omega}=Z_{\delta(\alpha)} .
$$

Если $\alpha \in \operatorname{Lim}$ Ord, то

$$
Y_{\alpha}=\operatorname{mix} \bigcup_{\beta<\alpha} Y_{\beta} \subset \operatorname{mix} \bigcup_{\beta<\alpha} Z_{\delta(\beta)} \subset \operatorname{mix} \bigcup_{\gamma<\delta(\alpha)} Z_{\gamma}=Z_{\delta(\alpha)} . \triangleright
$$

12.8. Следствие. Если $C$ - циклический подкласс $\mathbb{V}^{(B)}$ и $Y \uparrow \in C$ для всех $Y \subset C$, то $C=\mathbb{V}^{(B)}$.

$\triangleleft$ Допустим, $C \neq \mathbb{V}^{(B)}$. По теореме 12.7 существует наименьший ординал $\alpha$, для которого имеется элемент $x \in Z_{\alpha} \backslash C$. Если $\alpha=\beta+1$, то $x=Y \uparrow$ для некоторого подмножества $Y \subset Z_{\beta}$, и тогда из $Z_{\beta} \subset C$ вытекает $x \in C$. Если же $\alpha \in \operatorname{Lim}$ Ord, то $x=\sqcup P$ для некоторой максимальной антицепи $P \subset$ \% $\left(\bigcup_{\beta<\alpha} Z_{\beta}\right)$, и тогда из $\bigcup_{\beta<\alpha} Z_{\beta} \subset C$ вытекает $x \in \operatorname{mix} C=C$.

\section{ЛИТЕРАТУРА}

1. Гутман А. Е. Булевозначный универсум как алгебраическая система. І. Основные принципы // Сиб. мат. журн. 2019. Т. 60, № 5. С. 1041-1062.

2. Jech T. Set theory. The third millennium edition, revised and expanded. Berlin, etc.: SpringerVerl., 2003.

3. Lévy A. A hierarchy of formulas in set theory. Providence: Amer. Math. Soc., 1965.

4. Кусраев А. Г., Кутателадзе С. С. Введение в булевозначный анализ. М.: Наука, 2005.

5. Сикорский Р. Булевы алгебры. М.: Мир, 1969.

6. Jech T. Distributive laws // Handbook of Boolean algebras. Amsterdam; London: North-Holland, 1989. Chapter 8. P. 317-331.

7. Gutman A. E. Locally one-dimensional $K$-spaces and $\sigma$-distributive Boolean algebras // Sib. Adv. Math. 1995. V. 5, N 1. P. 42-48.

8. Бурбаки Н. Начала математики. Ч. 1. Основные структуры анализа. Книга 1. Теория множеств. М.: Мир, 1965.

9. Solovay R. M., Tennenbaum S. Iterated Cohen extensions and Souslin's problem // Ann. Math. 1971. V. 94, N 2. P. 201-245.

Поступила в редакцию 9 марта 2020 г.

После доработки 9 марта 2020 г.

Принята $к$ публикации 8 апреля 2020 2.

Гутман Александр Ефимович

Институт математики им. С. Л. Соболева СО РАН, пр. Академика Коптюга, 4, Новосибирск 630090;

Новосибирский государственный университет, ул. Пирогова, 1, Новосибирск 630090 gutman@math.nsc.ru 